\title{
Repositioning of Somatic Golgi Apparatus Is Essential for the Dendritic Establishment of Adult-Born Hippocampal Neurons
}

\author{
Sneha Rao, ${ }^{1}$ Gregory W. Kirschen, ${ }^{2}$ Joanna Szczurkowska, ${ }^{3}$ Adrian Di Antonio, ${ }^{1}$ Jia Wang, ${ }^{3}$ Shaoyu Ge, ${ }^{3}$ \\ and Maya Shelly ${ }^{3}$ \\ ${ }^{1}$ Program in Neuroscience, ${ }^{2}$ Medical Scientist Training Program, Stony Brook Medicine, and ${ }^{3}$ Department of Neurobiology and Behavior, State University \\ of New York-Stony Brook, Stony Brook, New York 11794
}

\begin{abstract}
New dentate granule cells (DGCs) are continuously generated, and integrate into the preexisting hippocampal network in the adult brain. How an adult-born neuron with initially simple spindle-like morphology develops into a DGC, consisting of a single apical dendrite with further branches, remains largely unknown. Here, using retroviruses to birth date and manipulate newborn neurons, we examined initial dendritic formation and possible underlying mechanisms. We found that GFP-expressing newborn cells began to establish a DGC-like morphology at $\sim 7 \mathrm{~d}$ after birth, with a primary dendrite pointing to the molecular layer, but at this stage, with several neurites in the neurogenic zone. Interestingly, the Golgi apparatus, an essential organelle for neurite growth and maintenance, was dynamically repositioning in the soma of newborn cells during this initial integration stage. Two weeks after birth, by which time most neurites in the neurogenic zone were eliminated, a compact Golgi apparatus was positioned exclusively at the base of the primary dendrite. We analyzed the presence of Golgi-associated genes using single-cell transcriptomes of newborn DGCs, and among Golgi-related genes, found the presence of STK25 and STRAD, regulators of embryonic neuronal development. When we knocked down either of these two proteins, we found Golgi mislocalization and extensive aberrant dendrite formation. Furthermore, overexpression of a mutated form of STRAD, underlying the disorder polyhydramnios, megalencephaly, and symptomatic epilepsy, characterized by abnormal brain development and intractable epilepsy, caused similar defects in Golgi localization and dendrite formation in adult-born neurons. Together, our findings reveal a role for Golgi repositioning in regulating the initial integration of adult-born DGCs.
\end{abstract}

Key words: adult-born neurons; early morphological integration; Golgi complex; hippocampus; STK25/STRAD

Significance Statement

Since the discovery of the continuous generation of new neurons in the adult hippocampus, extensive effort was directed toward understanding the functional contribution of these newborn neurons to the existing hippocampal circuit and associated behaviors, while the molecular mechanisms controlling their early morphological integration are less well understood. Dentate granule cells (DGCs) have a single, complex, apical dendrite. The events leading adult-born DGCs' to transition from simple spindle-like morphology to mature dendrite morphology are largely unknown. We studied establishment of newborn DGCs dendritic pattern and found it was mediated by a signaling pathway regulating precise localization of the Golgi apparatus. Furthermore, this Golgi-associated mechanism for dendrite establishment might be impaired in a human genetic epilepsy syndrome, polyhydramnios, megalencephaly, and symptomatic epilepsy.

\section{Introduction}

New dentate granule cells (DGCs) are continuously generated in a laminar zone beneath the granule cell layer of the adult hippocampus (Altman and Das, 1965; Eriksson et al., 1998; Zhao et

Received May 4, 2017; revised 0ct. 2, 2017; accepted 0ct. 29, 2017

Author contributions: S.R., S.G., and M.S. designed research; S.R., G.W.K., J.S., and A.D.A. performed research;

S.R., J.S., and J.W. contributed unpublished reagents/analytic tools; S.R. and G.W.K. analyzed data; S.R., S.G., and M.S. wrote the paper. al., 2008). Accumulating evidence, including work from our group, has revealed that, within 4 weeks, a newborn DGC undergoes full morphological maturation and completes functional

This work was supported by National Institutes of Health Grant NS084111 to M.S. and National Institutes of Health Grant 1F30MH110103 to Gregory W. Kirschen. We thank the Sneha Rao thesis committee members; Drs. David Talmage and Song-Hai Shi for suggestions and guidance on this project; Drs. David Talmage and Simon Halegoua on constructive comments on the manuscript; and Dr. Vivek Malhotra (Centre for Genomic Regulation, Barcelona, Spain), for providing a GRASP65 antibody. 
integration into the existing DGC circuit (Overstreet Wadiche et al., 2005; Ge et al., 2006, 2007; Zhao et al., 2006; Duan et al., 2007; Kumamoto et al., 2012). Newly generated DGCs exhibit a spindle-like structure in the neurogenic zone. Within $\sim 2$ weeks after birth, the surviving newborn cells develop a granule cell-like morphology with a single primary dendrite, exhibiting a complex morphology with secondary, tertiary, and further branches, pointing to the molecular layer (Espósito et al., 2005; Zhao et al., 2006). However, the establishment of the single dendrite is preceded by extensive neurite remodeling, generation followed by elimination, of neurites in the neurogenic zone. In contrast to extensive studies of subsequent dendritic branching, pruning, synapse formation and spine morphogenesis (Zhao et al., 2008), how and when a newborn neuron initially forms the mature granule cell-like morphology, and the underlying mechanisms, remain poorly understood.

The Golgi apparatus shows dynamic localization in the soma during neuronal development and has been implicated in differential regulation of neurite growth (Horton et al., 2005; de Anda et al., 2005; Ye et al., 2007), and apical and basal dendrite development in embryonic pyramidal neurons (Horton et al., 2005; Ye et al., 2007; Matsuki et al., 2010). Further evidence showed that the Golgi apparatus operates as a signaling platform organized by the Golgi matrix proteins that enable asymmetric Golgi complex positioning (Barr and Short, 2003; Bivona et al., 2003; Short and Barr, 2003; Ríos et al., 2004), to mediate cell development, migration, or polarization (Kupfer et al., 1982; Kupfer and Dennert, 1984; Bivona et al., 2003; Ríos et al., 2004; Yadav et al., 2009). In developing neurons, such Golgi signaling scaffolds might enable asymmetric Golgi subcellular localization (Kupfer et al., 1982; Kupfer and Dennert, 1984) to promote directed membrane supply (Horton et al., 2005) or serve as acentrosomal microtubule nucleation sites (Ori-McKenney et al., 2012), necessary for polarized neurite formation, growth, or maintenance. We therefore asked whether the Golgi apparatus could, through its dynamic positioning, regulate the neuritic rearrangement of newborn DGCs during the establishment of the dendritic morphology.

Here, we examined dendrite formation and establishment at an early stage of adult-born DGC integration when a single dendrite is specified, and explored the underlying mechanisms. We used a retroviral labeling approach to mark and genetically manipulate newborn DGCs in the adult brain (Gu et al., 2011; Kumamoto et al., 2012). First, we analyzed dendrite establishment of newborn DGCs and determined its timeline. Interestingly, we found that, during the establishment of a single dendrite, there was robust redistribution of the subcellular Golgi apparatus in newborn DGCs, which coincided with neurite remodeling. Furthermore, our analysis of single-cell transcriptomes from doublecortin (DCX)-positive adult-born DGCs (obtained from Gao et al., 2017), and our immunohistochemical examinations, revealed robust expression of the polarity genes, Sps1/Ste20-related kinase-1 STK25 (also known as YSK1, Sok1) (Osada et al., 1997; Preisinger et al., 2004) and the adaptor protein STE20-related pseudokinase (STRAD) (Boudeau et al., 2004), along with the Golgi matrix protein GM130 (Nakamura et al., 1995; Puthenveedu et al., 2006), crucial regulators of axon and dendrite polar-

The authors declare no competing financial interests.

Correspondence should be addressed to Dr. Maya Shelly, Department of Neurobiology and Behavior, Life Sciences Building, Room 530, State University of New York-Stony Brook, Stony Brook, NY 11794. E-mail: maya.shelly@stonybrook.edu

DOI:10.1523/JNEUROSCI.1217-17.2017

Copyright $\odot 2018$ the authors $\quad 0270-6474 / 18 / 380632-17 \$ 15.00 / 0$ ization during embryonic neuronal development in a manner that depended on Golgi localization (Preisinger et al., 2004; Matsuki et al., 2010). By manipulating these candidate Golgipositioning genes, we found that STK25 and STRAD enabled polarized Golgi localization and played a critical role in dendrite establishment during adult-born DGC initial integration.

\section{Materials and Methods}

All surgeries and experimental procedures were approved by the Stony Brook University Animal Use Committee and followed the guidelines of the National Institutes of Health.

Antibodies and materials. The following antibodies were used for immunohistochemistry: anti-GFP (Rockland Immunochemicals or Aves Labs), anti-dTomato (Rockland), anti-MAP2 (Abcam), antiAnkG (University of California-Davis/National Institutes of Health NeuroMab Facility), anti-GRASP65 (Abcam, Novus Biologicals, or a kind gift from Dr. Vivek Malhotra, Centre for Genomic Regulation, Barcelona, Spain), anti-STK25 (Novus Biologicals or Santa Cruz Biotechnology), anti-STRAD (Novus Biologicals or Santa Cruz Biotechnology), anti-LKB1 (Novus Biologicals), and anti-Na,K-ATPase (Developmental Studies Hybridoma Bank, University of Iowa). Fluorescently conjugated secondary antibodies were from Invitrogen or Jackson ImmunoResearch Laboratories. The following antibodies were used for immunoprecipitation or immunoblotting: anti-GFP (Roche Diagnostics), anti-dTomato (Rockland), anti- $\beta$-actin (Cell Signaling Technology), anti-FLAGM2 antibody (Sigma-Aldrich), anti-STK25 (Santa Cruz Biotechnology), anti-STRAD (Novus), and anti-LKB1 (Acris-OriGene Technologies). HRP-conjugated secondary antibodies were from Jackson ImmunoResearch Laboratories. Doxycycline (DOX) was from Sigma-Aldrich.

Retroviral shRNA and expression constructs and retrovirus production. For noninducible downregulation, two specific short hairpin oligomers for STK25 (shSTK25-245 and shSTK25-665) or STRAD (shSTRAD-1060 and shSTRAD-325) were prepared and cloned into the PUEG retroviral vector previously described (Duan et al., 2007). The shRNA target sequences were selected using the RNAi Codex tool from Cold Spring Harbor Laboratory and verified using the Web-based program siRNA at Whitehead. The shRNA sequences were cloned using the BglII and XbaI sites of pUEG, to generate pUEG-shSTK25 or pUEG-shSTRAD, under the hU6 promoter. The vector also expressed GFP under the EF1 $\alpha$ promoter. The sequences of oligomers were the following: shSTK25-245, 5' -CTCTAGAAAAAAAGCCGCATTGGCAAAGGCTCATTCAAGCTT CAATGAGCCTTTGCCAATGCGGCGGATCCTCGTCCTTTCCAC-3'; shSTK25-665, 5'-CTCTAGAAAAAAAGGCTCACAGACACACAAATCA ACAAGCTTCTTGATTTGTGTGTCTGTGAGCCGGATCCTCGTCCT TTCCAC-3'; shSTRAD-325, 5'-CTCTAGAAAAAAAGAATGAGATG GTGACATTCTTGCAAGCTTCCAAGAATGTCACCATCTCATTCGGA TCCTCGTCCTTTCCAC-3'; shSTRAD-1060, 5'-CTCTAGAAAAAAA GAACTTTGTGGAACAGTGCCTTCAAGCTTCAAGGCACTGTTCCA CAAAGTTCGGATCCTCGTCCTTTCCAC-3' ${ }^{\prime}$; shGM130-seq 1, 5' -CTC TAGAAAAAAAGCGGCCATGGTGGCATTCTTTAACAAGCTTCTTA AAGAATGCCACCATGGCCTCGGATCCTCGTCCTTTCCAC-3'; and shGM130-seq2, 5' -CTCTAGAAAAAAAGATGAGAACATGGAGATCA CCACAAGCTTCTGGTGATCTCCATGTTCTCATCGGATCCTCGTC CTTTCCAC-3'. PCR was performed on the empty pUEG plasmid with the forward oligomer pSIREN-5F BglII, 5'-GAATTGAAGATC TGGG CAGGAAGAGGGC-3'

For DOX-inducible downregulation, the pSIREN-RetroQ-TetP vector (Clontech Laboratories) was modified by linking the tTS suppressor element to EGFP via a P2A tag; thus, EGFP was expressed constitutively under the ubiquitin promoter from the same construct. The shRNA was expressed under the Tet-on TRE mod/u6 promoter. The shRNA sequences were cloned using the BamHI and EcoRI sites of pSIREN to generate pSIREN-shSTRAD or pSIREN-shSTK25 constructs. The following shRNA oligomer pairs with BamHI and EcoRI overhangs were used: shSTK25-245, 5'-GATCCGCCGCATTGGCAAAGGCTCATTT TCAAGAGAAATGAGCCTTTGCCAATGCGGTTTTTTACGCGTG-3' and $5^{\prime}$-AATTCACGCGTAAAAAACCGCATTGGCAAAGGCTCATTT 
CTCTTGAAAATGAGCCTTTGCCAAGCGGCG-3'; shSTK25-665, 5' GATCCGGCTCACAGACACACAAATCAATTCAAGAGATTGATTTG

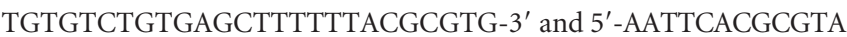
AAAAAGCTCACAGACACACAAATCAATCTCTTGAATTGATTTGT GTGTCTGTGAGCCG-3'; shSTRAD-325, 5'-GATCCGAATGAGATG GTGACATTCTTGTTCAAGAGACAAGAATGTCACCATCTCATTTT TTTTTCGCGA-3' and 5' -AATTCTCGCGAAAAAAAAATGAGATGG TGACATTCTTGTCTCTTGAACAAGAATGTCACCATCTCATTCG-3'; and shSTRAD-1060, 5' -GATCCGAACTTTGTGGAACAGTGCCTTTT CAAGAGAAAGGCACTGTTCCACAAAGTTTTTTTTTCGCGA-3' and 5'-AATTCTCGCGAAAAAAAAACTTTGTGGAACAGTGCCTTTCTC TTGAAAAGGCACTGTTCCACAAAGTTCG-3'. Efficiency of downregulation was examined upon coexpression of the shRNA constructs together with the targeted STK25 or STRAD expression vectors in HEK293 cells, by immunoblotting (see below).

To generate retroviral expression constructs for mouse STK25 and STRAD, the mouse STK25 and STRAD cDNAs were synthesized from total RNA of 4- to 6-week-old C57BL/6 mouse brain by reverse transcription, and cloned into DOX-inducible retroviral construct, pTet (Kumamoto et al., 2012). The sequence of mouse STK25 or STRAD was PCR-amplified from the cDNA, using oligonucleotide primers: STK25, 5'-ATGGCTCACCTCCGGGGCTTC-3' and 5' ${ }^{\prime}$-TCAGCGGGTAGATG TCAGGTG-3' , corresponding to mouse STK25 (NM_021537); STRAD, $5^{\prime}$-ATGGTGAGCAAGGGCGAGGA- ${ }^{\prime}$ and $5^{\prime}$-TCAGAACTCCCAGTC ATCCAC-3', corresponding to mouse STRAD (NM_001252448). The STK25 and STRAD cDNAs were fused to EGFP or dTom, and cloned into the pTet vector. The STRAD mutant form STRAD-PMSE-D180, fused to dTomato, was generated using standard PCR procedures.

Retroviral suspensions were prepared by cotransfection of modified replication-defective Murine Moloney Leukemia virus constructs (Ge et al., 2006; Kumamoto et al., 2012), with VSVG and GP constructs into HEK-293 cells. Viruses were concentrated from the viral supernatant by ultracentrifugation and suspended in sterile PBS for stereotaxic injections.

Retroviral stereotaxic injections. Stereotaxic injections were performed in 4- to 6-week-old C57BL/6 wild-type mice (Charles River) per protocols approved by the Institutional Animal Care and Use Committees at Stony Brook University, and as previously described (Gu et al., 2011). Following anesthesia, animals were mounted on a stereotaxic frame (Stoelting), and holes were drilled in the skull at the following coordinates: anterior dentate gyrus, measurement from bregma: anteroposterior $=-2 \mathrm{~mm}$, mediolateral $= \pm 1.6 \mathrm{~mm}$. Posterior dentate gyrus, measurement from bregma: anteroposterior $=-3 \mathrm{~mm}$, mediolateral $= \pm 2.6 \mathrm{~mm}$.

Retroviruses were injected bilaterally into the drilled sites. Ventral depth was measured by placing the needle tip on the midline between the 2 anterior drill sites, and measuring as follows: anterior dentate gyrus: dorsoventral $=-2.5 \mathrm{~mm}$. Posterior dentate gyrus: dorsoventral $=-3.2$ $\mathrm{mm}$. For DOX-induced expression, DOX (Sigma-Aldrich) in 5\% sucrose was administered orally via drinking water at a concentration of $2 \mathrm{mg} / \mathrm{ml}$ starting at the appropriate time point, for the duration of the experiment.

Retroviruses expressing GFP or dTomato, pUX-GFP or pUX-dTomato, under the ubiquitin promoter, as described previously (Ge et al., 2006; Gu et al., 2011), were used to label adult-born neurons for developmental time course analyses, and as an internal control for all genetic manipulations, in this study.

Immunohistochemistry. Mice were perfused with $4 \%$ PFA and the brains isolated, at 5, 7, 10, or $14 \mathrm{~d}$ postretroviral injections (dpi). Following $24 \mathrm{~h}$ equilibration in $30 \%$ sucrose, the brains were coronally sectioned at the level of hippocampi and processed for immunohistochemistry. For morphological analyses, brains were sectioned on a sliding microtome at $60 \mu \mathrm{m}$, and processed as free-floating sections. Floating brain slices were permeabilized with $0.3 \%$ Triton X-100, blocked in $10 \%$ normal donkey serum and $0.3 \%$ Triton $\mathrm{X}-100$, and were incubated for $24 \mathrm{~h}$ in the primary antibody at $4^{\circ} \mathrm{C}$. The sections were immunostained with antibodies for GFP and dTomato (Rockland), for clear visualization of the neuritic arbor of the virus-infected cells. Golgi apparatus was labeled with antibody for the Golgi-cisternae stacking protein, GRASP65 (Abcam). For immunolabeling with the dendritic marker MAP2 (Abcam), brains were sectioned at $30 \mu \mathrm{m}$ using cryostat (Leica), mounted onto glass slides, and subjected to antigen-retrieval procedure before immunostaining. Slide-mounted cryosections were permeabilized with $0.3 \%$ Triton X-100 and subjected to antigen retrieval (Antigen retrieval buffer, Wako Chemicals) for $10-30 \mathrm{~min}$, at $\mathrm{pH} 6.0$, at $90^{\circ} \mathrm{C}-95^{\circ} \mathrm{C}$. Following blocking in $10 \%$ donkey serum and $0.6 \%$ Triton X-100, slides were incubated with MAP2antibody, for $36 \mathrm{~h}$ at $4^{\circ} \mathrm{C}$, and processed for secondary antibody labeling. The slices were mounted in Fluomount G (Southern Biotechnology).

Image acquisition and analyses. For the analysis of the morphology of DGC neurons, brains were perfused and dissected at 5, 7, 10, and $14 \mathrm{dpi}$. For morphometric analysis, confocal images (30 6- $\mu$ m-thick $z$ stacks) of isolated neurons were acquired with a $40 \times$ or $60 \times($ NA 1.43) oil-immersion objective lens, on the Fluoview FV1000 confocal microscope (Olympus). For quantification of neurite number, 3D images of entire dendritic arbors were reconstructed from $z$ series stacks of the confocal images using IMRS 7.3.1 software (Bitplane), and 3D traces of the neuritic arbor of representative fluorescently labeled DGCs, were generated. 2D traces of the GRASP65 fluorescence in individual GFP-labeled DGCs, including the traces of the cell contour and the neuritic arbor using GFP fluorescence, were generated using ImageJ software, to examine Golgi localization.

Our quantitative analysis of neurite number included all primary neurites directly extending from the soma, oriented to the granule cell layer, the molecular layer, or the hilus. To quantify Golgi localization, each infected neuron in 2D images was divided into four quadrants: apical, basal and two lateral. Mean fluorescence intensity of GRASP65 of each quadrant, relative to total GRASP65 fluorescence (\% total fluorescence), was calculated, following subtraction of background GRASP65 signal (ImageJ).

STK25, STRAD, and Na, K-ATPase signals were identified and related to their neurons of origin by examining 3D, $60 \mu \mathrm{m} z$-stacked images using Imaris Scientific 3D/4D Processing and Analysis Software (Bitplane). Regions of interest for each cell were defined, and $3 \mathrm{D}$ reconstructions of each channel were created using the mixed model rendering function.

Transfection, immunoprecipitation, and immunoblotting. HEK-293 cells were used for examination of biochemical associations of STK25 with the STRAD/LKB1/MO25 complex and GM130, and the effects of STRAD-PMSE-D180 expression on these associations. These cells were also used to examine efficiency of shRNA-mediated downregulation. For these biochemical examinations, GFP- or dTom-fused STK2 5 or STRAD were cloned into the mammalian expression vector pCDNA3. For these studies, we also generated expression constructs for mouse LKB1 or GM130 with the following primers: LKB1, 5' -ATGGTGAGCAAGGGCGAGGA-3' and $5^{\prime}$-TCAGCGGGTAGATGTCAGGTG-3', corresponding to mouse LKB1 (NM_011492); GM130, 5'-ATGTGGCCCCCCCGCTTCCCC-3' and $5^{\prime}$-TTATACAACCATGATCTTCACCTCGTCGTTCTC-3', corresponding to mouse GM130 (NM_133852). The LKB1 was fused to EGFP or dTom, and the GM130 was fused to the Flag sequence. For these biochemical examinations, we also used rat constructs for LKB1, STRAD, or MO25, cloned into pCDNA3, as previously described (Shelly et al., 2007).

HEK-293 cells were grown in DMEM supplemented with 10\% FBS, and were transfected with $2 \mu \mathrm{g}$ of plasmid DNA using calcium-phosphate method. Efficiency of downregulation was verified by cotransfection of the shRNA constructs together with the targeted expression constructs, and cells were either treated with DOX or left untreated. At $48 \mathrm{~h}$ after transfection, the cells were lysed and processed for immunoblotting or immunoprecipitation.

For immunoprecipitation, $1-2 \mu \mathrm{g}$ of primary antibody with $20 \mu \mathrm{l}$ of protein $\mathrm{G}$ agarose beads (Roche) was added to the cell lysate and incubated under constant agitation for $4-16 \mathrm{~h}$, at $4^{\circ} \mathrm{C}$. Immunoblotting was performed using standard detection techniques. Quantification of the biochemical associations or the efficiency of shRNA-mediated downregulation was performed using ImageJ software.

Statistics and data analysis. Process number and Western blot data were quantified with Microsoft Excel or MATLAB (The MathWorks). Student's two-tailed, unpaired $t$ test, Kolmogorov-Smirnov test, or $\chi^{2}$ test was used to determine statistical significance at $p<0.05$. Transcriptome data were obtained from Gao et al. (2017) and analyzed with Microsoft Excel. Gene expression patterns were represented by heat maps generated using MATLAB (The MathWorks). 

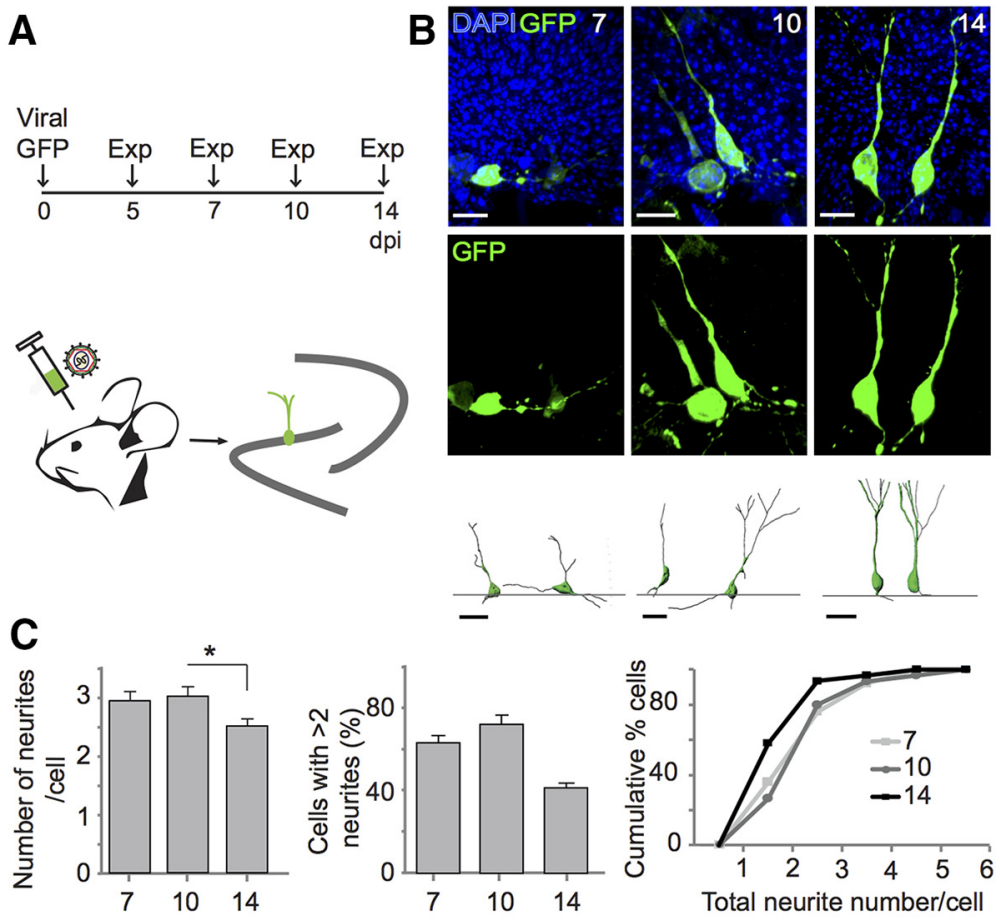

D
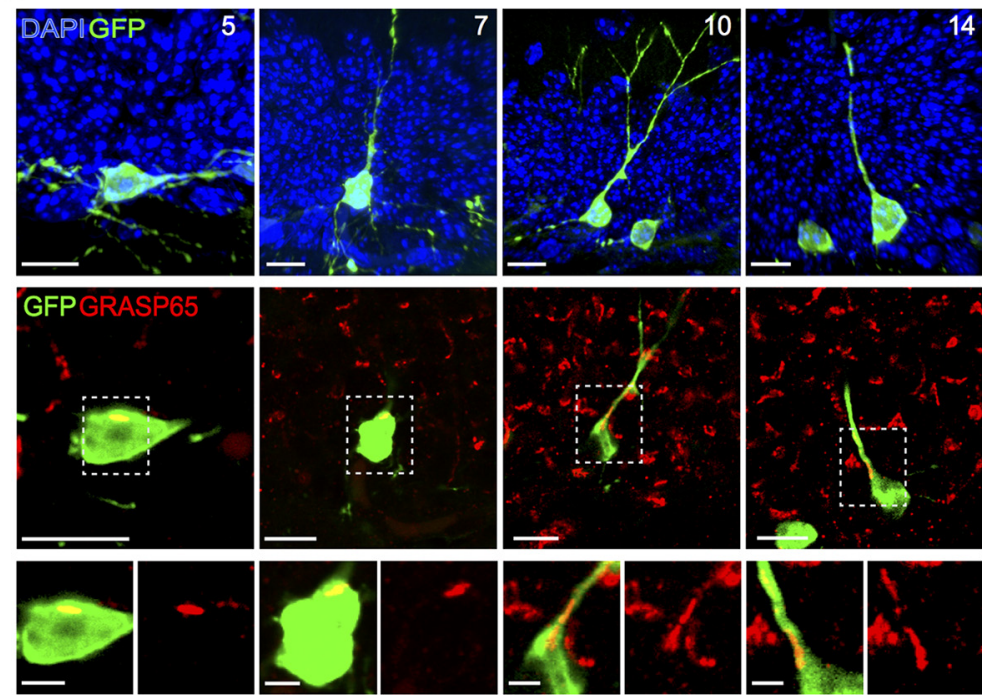

E

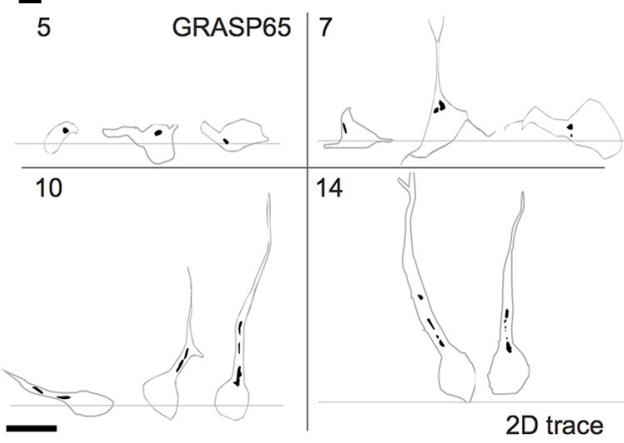

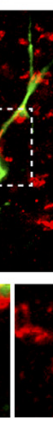

$\mathrm{F}$

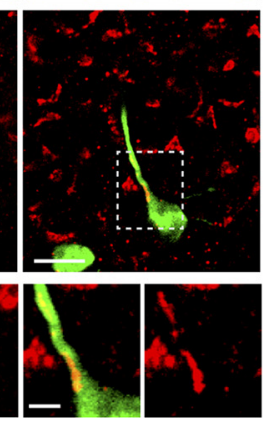

=no neurites -primary neurite

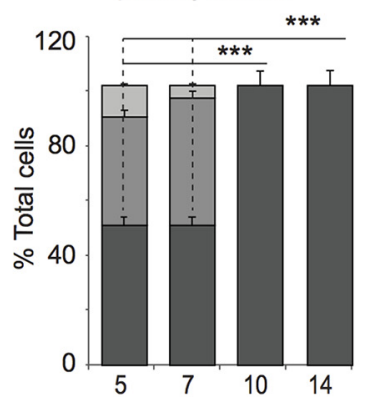
»non-primary neurite

Figure 1. Initial integration of adult-born DGCs is associated with Golgi repositioning and neurite remodeling. $\boldsymbol{A}$, Schematic depiction of the paradigm for retroviral injection of GFP retrovirus (pUX-GFP) into dentate gyrus of wild-type 4- to 6-week-old mice, and examination at 5, 7, 10, or $14 \mathrm{dpi}$. $\boldsymbol{B}$, Top, Confocal images ( $60 \mu \mathrm{m} z$ stacks) of GFP fluorescence of representative GFP retrovirus (pUX-GFP)-infected adult-born DGCs at 7, 10, and $14 \mathrm{dpi}$. Granule cell layer (GCL) visualized by DAPI staining. Scale bar, $10 \mu \mathrm{m}$. Bottom, 3D traces (Imaris, Bitplane) of the neuritic arbor from confocal images of representative GFP-labeled DGCs at 7, 10,

\section{Results}

The establishment of the dendritic pattern of adult-born DGCs is associated with Golgi repositioning A mature DGC exhibits a single dendrite, with multiple secondary, tertiary, and further branches in the molecular layer, forming a laminar activity input layer (Dudek and Sutula, 2007). One would expect that, during dendritic integration into an existing network, the newborn DGCs would undergo extensive morphological changes. However, in contrast to the well-studied dendritic pruning and spine formation, the initial morphological establishment of young adult-born DGCs and the underlying mechanisms remain poorly understood. In this study, we examined dendritic morphological establishment of adult-born DGCs and possible underlying mechanisms.

We first examined dendrite formation in newborn DGCs during their initial integration. We used a retroviral approach to label dividing neural progenitors and

\footnotetext{
and 14 dpi. Horizontal line indicates the bottom of the GCL.C, Left, Quantification of average total number of neurites per cell at 7, 10, and 14 dpi in GFP-labeled DGCs. The quantitative analysis included all primary neurites directly extending from the soma, oriented to the $\mathrm{GCL}$, the ML, or the hilus ( $7 \mathrm{dpi}, 25$ cells; $10 \mathrm{dpi}, 30$ cells; $14 \mathrm{dpi}, 31$ cells; from at least 3 mice per time point; $p<0.05$, Student's two-tailed $t$ test, $p=0.018$, power $=0.56)$. Middle, Quantification of the average percentage of GFP-labeled DGCS that had $>2$ neurites at 7, 10, and $14 \mathrm{dpi}$. Right, Cumulative percentage plots for total number of neurites per cell at 7, 10, and $14 \mathrm{dpi}$. Same dataset was used for all quantifications in C. D, Confocal images $(60 \mu \mathrm{m} \mathrm{z}$ stacks) of Golgi labeling in representative GFP retrovirusinfected DGCs at 5, 7, 10, and 14 dpi, immunostained for the Golgi apparatus marker GRASP65. GCL visualized by DAPI staining. Scale bar, $10 \mu \mathrm{m}$. Bottom, Higher-magnification images (of boxed regions, top) showing Golgi localization in the soma or to the primary neurite pointing to the ML. Scale bar, 4 $\mu \mathrm{m} . \boldsymbol{E}, 2 \mathrm{D}$ traces of GRASP 65 fluorescence in GFP-labeled DGCs from confocal images of representative GFP-labeled DGCs immunostained for GRASP65 at 5, 7, 10, and 14 dpi. The cell contour and neuritic arbor were traced using GFP fluorescence. Horizontal line indicates the bottom of the GCL. Scale bar, 10 $\mu \mathrm{m}$. $\boldsymbol{F}$, Quantification of percentage of cells with different intracellular Golgi localization in GFP-labeled DGCs immunostained for GRASP65 at 5, 7, 10, and 14 dpi, categorized as follows: "no neurites," localization in the soma in neurons with no visible neurites; "nonprimary neurite," localization at the base of a single neurite that is not the primary neurite pointing to the ML; and "primary neurite," localization to the base of a primary neurite pointing to the $\mathrm{ML}$ in neurons that might also have other neurites. These data demonstrate that, during development, the Golgi shows dynamic repositioning to the primary neurite, which points to the ML ( $5 \mathrm{dpi}, 18$ cells; $7 \mathrm{dpi}, 22$ cells; $10 \mathrm{dpi}, 24$ cells; $14 \mathrm{dpi}, 19$ cells; from at least 3 mice per time point; $\chi^{2}$ test, comparing number of cells with or without Golgi positioning to the primary neurite at different time points, $p<0.05) .{ }^{*} p \leq 0.05,{ }^{* * *} p \leq 0.001$.
} 
their progeny, to follow their maturation over a 2 week period (Gu et al., 2011; Kumamoto et al., 2012) (Fig. 1A). Qualitative analysis of typical images of GFP-expressing neurons and samples of reconstructed cells (Fig. $1 B$ ) at 7, 10, and 14 days post injection (dpi) revealed that, between 7 and $10 \mathrm{dpi}$, immature neurons formed multiple neurites oriented to the hilus or the granule cell layer. By 10 dpi, most cells also formed a primary neurite pointing to the molecular layer, which was likely to become the apical dendrite. By 14 dpi, cells had undergone substantial remodeling of their neurites, and only the primary dendrite, together with one or two thin hilar neurites, remained (Fig. $1 B$ ). Quantification of the total number of neurites in each cell (Fig. 1C, left) and cumulative distribution of the percentage of cells with varying number of neurites (Fig. $1 C$, right) revealed a higher number of neurites per cell at 7 and $10 \mathrm{dpi}$, compared with $14 \mathrm{dpi}$. Furthermore, quantification of the percentage of cells with $>2$ neurites (Fig. $1 C$, middle) showed that $\sim 64 \%$ and $73 \%$ of neurons at 7 and $10 \mathrm{dpi}$, respectively, had $>2$ neurites, compared with only $40 \%$ at $14 \mathrm{dpi}$. The quantitative analysis included all primary neurites directly extending from the soma, oriented to the granule cell layer (GCL), the molecular layer, or the hilus. Thus, newborn DGCs form multiple neurites by $7 \mathrm{dpi}$, most of which are eliminated by $14 \mathrm{dpi}$. The morphology displayed at $14 \mathrm{dpi}$ is comparable with the fully mature morphology observed at $28 \mathrm{dpi}$ (data not shown), with only a single primary dendrite. We conclude that the initial integration of newborn DGCs is associated with formation and elimination of extraneous neurites and generation of a single primary dendrite in the granule cell layer.

Asymmetric localization of the Golgi apparatus was shown to regulate dendrite specification in developing embryonic neurons (Jareb and Banker, 1997; de Anda et al., 2005; Horton et al., 2005; Ye et al., 2007; Matsuki et al., 2010; Tanabe et al., 2010). We analyzed Golgi distribution in newborn DGCs by immunostaining GFP-retroviral labeled cells for the Golgi-cisternae stacking protein GRASP65, at 5, 7, 10, and 14 dpi (Fig. 1D). Analysis of 2D traces of the Golgi in individual GFP-labeled neurons demonstrated that, at $14 \mathrm{dpi}$, a time when a single primary dendrite is already specified, the Golgi was predominantly localized to the base and proximal shaft of the primary dendrite (Fig. $1 E, F$ ). At this stage, we did not detect GRASP65 signal in other parts of the soma. When analysis was extended to 5 and $7 \mathrm{dpi}$, we observed that the Golgi demonstrated compact, yet dynamic, subcellular localization (Fig. 1D-F). Our analysis showed that, at 5-7 dpi, the Golgi was associated with the base of a single neurite (Fig. 1E,F; $>80 \%$ cells; "primary" or "nonprimary" neurite). At this stage, some newborn DGCs form a primary neurite in the granule cell layer which points toward the molecular layer, representing the future apical dendrite. At 5 and 7 dpi, if this primary neurite had formed, the Golgi was already preferentially localized to its base (Fig. 1E,F; 50\% cells; "primary" neurite). By 10 dpi, the Golgi exclusively ( $100 \%$ cells) localized to the base of the primary neurite (Fig. $1 E, F$ ), like that found at $14 \mathrm{dpi}$.

Together, our analyses showed that, right after birth, newborn DGCs experience a phase of extensive neurite remodeling that is accompanied by the repositioning of the Golgi complex to the base of the primary dendrite. This finding supports the idea that the repositioning of the Golgi apparatus may be involved in the dendritic establishment of newborn DGCs.

\section{Knockdown of the Golgi apparatus-related protein STK25 impaired dendrite establishment in adult-born DGCs} To assess the possible role of Golgi complex localization in dendrite establishment, we analyzed single-cell transcriptomes of young adult-born DGCs (Gao et al., 2017) and found that many DCX-positive DGCs expressed most known Golgi-associated genes (for review, see Yadav and Linstedt, 2011) (Fig. 2A). Particularly, we found that transcripts for the Golgi-polarity gene STK25 (Sps1/Ste20-related kinase 1, also known as YSK1, Sok1) (Osada et al., 1997; Preisinger et al., 2004) were expressed in DCX-positive adult-born DGCs (Fig. 2A). We quantified the expression of STK25 in newborn DGCs in wild-type 4- to 6-weekold mouse hippocampus, by coimmunolabeling with DCX to mark adult-born neurons. Our examination showed that nearly all DCX-positive cells express STK25 (Fig. 2B). STK25 was shown to be a crucial regulator of Golgi positioning and cell polarization during embryonic neuronal development (Preisinger et al., 2004; Matsuki et al., 2010). Because our observations showed that the Golgi apparatus is preferentially repositioned to the primary dendrite early in integrating DGCs (Fig. $1 D-F$ ), we asked whether STK25 is involved in Golgi localization and in the regulation of dendrite establishment in adult-born DGCs.

To determine the role of STK25 in Golgi complex repositioning and in dendrite establishment, we downregulated its expression in newborn DGCs with a retroviral mouse STK25-shRNA vector (Duan et al., 2007), pUEG-shSTK25, which expressed shRNA and EGFP under separate promoters (Fig. 2C). This vector efficiently downregulated the expression of targeted mouse STK25 coexpressed in HEK-293 cells (data not shown). High titer retrovirus for pUEG-shSTK25 was coinjected into the dentate gyrus of wild-type 4- to 6-week-old mouse hippocampus, together with dTom control virus (pUX-dTom) that served as an internal control. We immunolabeled pUEG-shSTK25-infected adult-born DGCs with antibodies against STK25, and examined the level of STK25 expression upon knockdown with pUEGshSTK25 compared with control neighboring adult-born DGCs immunolabeled with DCX. These experiments showed efficient knockdown of STK25 expression in pUEG-shSTK25-infected neurons compared with control DCX-positive neurons (Fig. 2C). We examined Golgi localization in pUEG-shSTK25-infected DGCs by GRASP65 immunostaining (Fig. 2D-F) at $14 \mathrm{dpi}$, a time when Golgi has exclusively repositioned to the primary dendrite and dendrite establishment is completed in control newborn DGCs (Fig. 1). Quantification of GRASP65 expression in adult-born DGCs by coimmunolabeling with DCX showed that GRASP65 was expressed nearly in all DCX-positive adult-born DGCs (Fig. 2B). Images of adult-born DGCs were acquired using EGFP or dTom fluorescence for shRNA- or control-infected neurons, respectively. As we showed above (Fig. $1 D-F$ ), at $14 \mathrm{dpi}$, the Golgi was preferentially localized to the base of the primary dendrite in control neurons. In stark contrast, in pUEG-shSTK25infected neurons (Fig. 2D,E), the Golgi was dispersed throughout the soma and was no longer exclusively localized to the base of the primary dendrite. To quantify the dispersion of the Golgi upon STK25 downregulation, we divided each infected neuron in the $2 \mathrm{D}$ projection images into four quadrants (apical, basal and two lateral), with the apical quadrant oriented to the molecular layer and the basal quadrant oriented to the hilus (Fig. $2 F$ ), and calculated the mean fluorescence intensity of GRASP65 in each quadrant relative to total GRASP65 fluorescence (percent total fluorescence). Consistent with our observations (Fig. 2D,E), the quantitative analysis showed that the GRASP65 signal was largely restricted to the apical quadrant in control cells. However, in STK25-shRNA-infected cells, the GRASP65 signal was uniformly distributed throughout the four quadrants, indicating Golgi dispersion (Fig. 2F). To examine whether the Golgi-secretory function is affected upon STK25 knockdown and the observed Golgi 
A

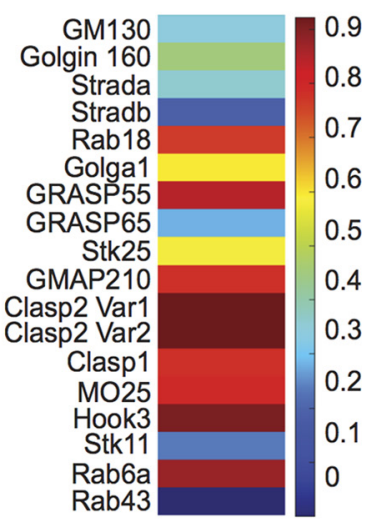

B
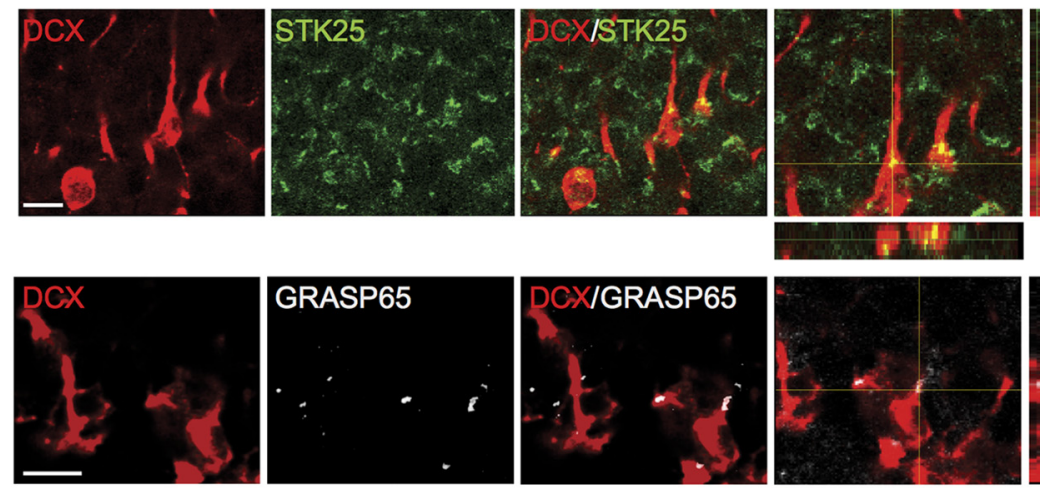

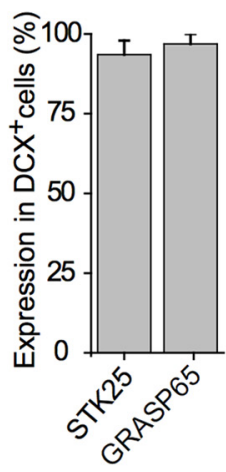

(Analyzed from Gao et al., 2016)

C

pUEG : u6-shSTK25-EF1a- GFP
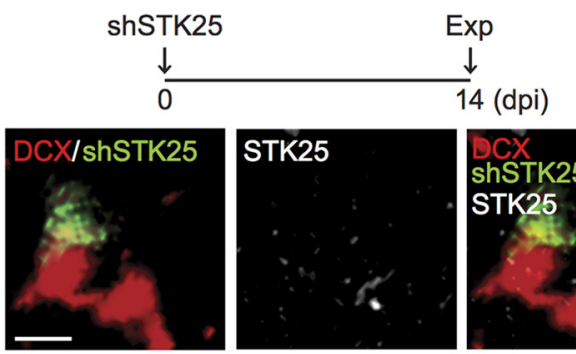

E
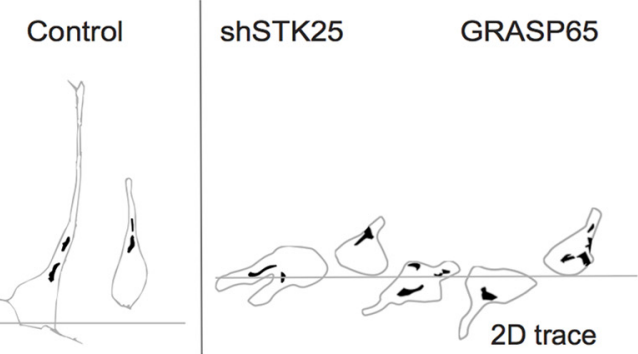

$\mathbf{F}$
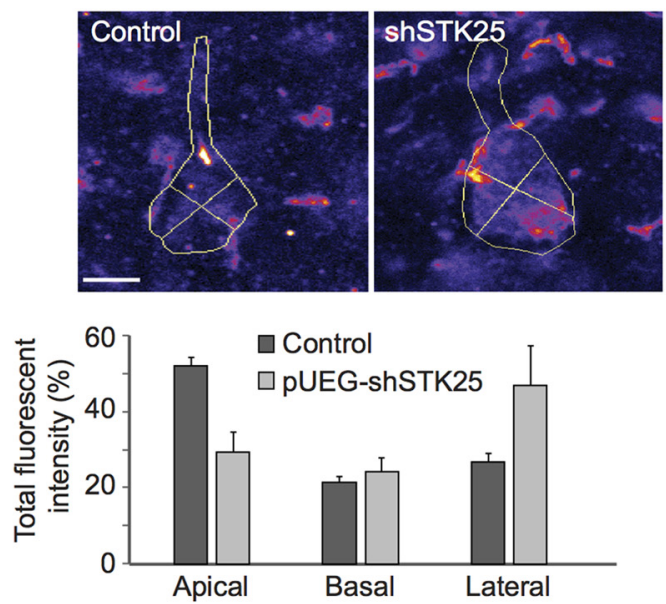

D
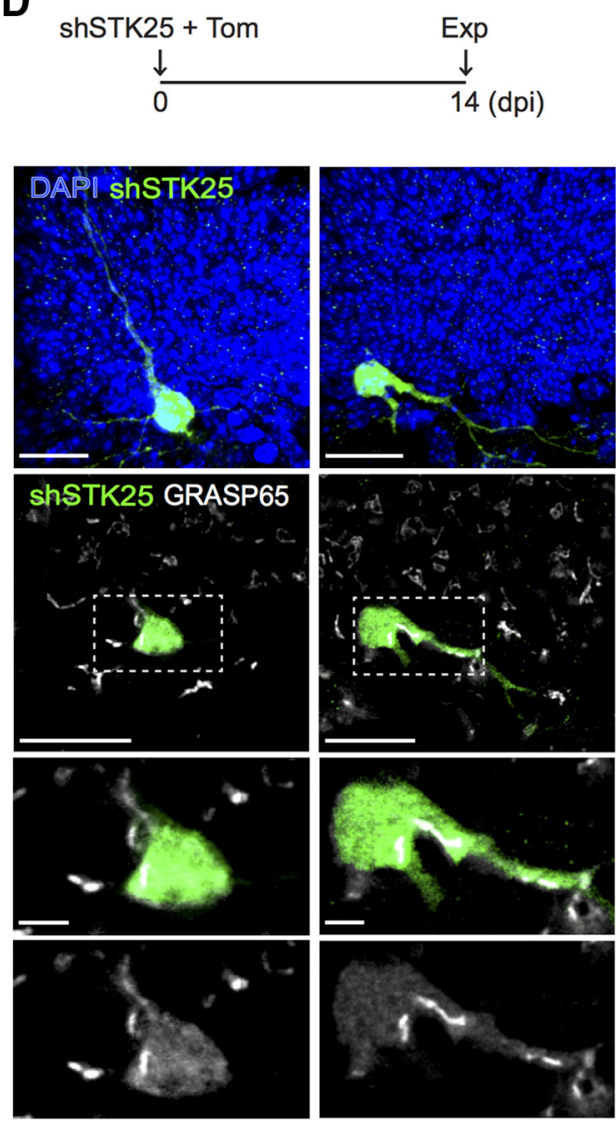

G
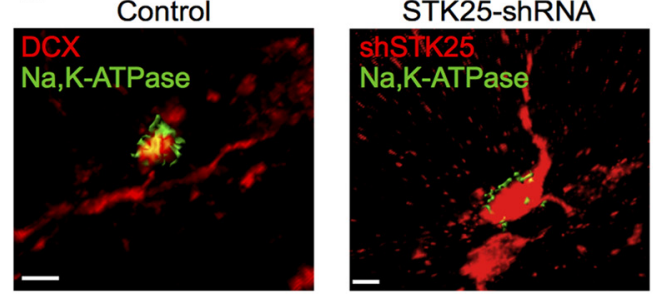

Figure 2. STK25 regulates Golgi positioning during early DGC integration. $\boldsymbol{A}$, Heatmap of fraction of DCX-positive DGCs from the mouse dentate gyrus, expressing various Golgi-associated genes, analyzed from Gao et al. (2017) (Gene Expression Omnibus accession \#GSE75901). For quantification of specific protein expression in DCX-positive adult-born DGCs, see $\boldsymbol{B}$; and Figure 4A. $\boldsymbol{B}$, Left, Confocal images of representative adult-born DGCs from wild-type 4- to 6-week-old mouse hippocampus immunolabeled with DCX and STK25 or GRASP65. Coexpression was confirmed by 3-plane analysis. Scale bar, $10 \mu \mathrm{m}$. Right, Quantification of the average percentage of DCX-positive adult-born DGCs that express STK25 or GRASP65, determined based on coimmunolabeling with DCX and either STK25 or GRASP65 (STK25, 80 cells; GRASP65, 67 cells; from 4 separate mice). Nearly all DCX-positive cells express STK25 and GRASP65. C, Top, Schematic depiction of the retroviral mouse STK25-shRNA vector, pUEG-shSTK25, with shRNA and EGFP expression under the u6 or EF1 $\alpha$ promoters, respectively, and paradigm for coinjection of (Figure legend continues.) 
dispersion, we tested the cell surface expression of the $\mathrm{Na}, \mathrm{K}$ ATPase in adult-born DGCs infected with pUEG-shSTK25, at 14 dpi. The Na,K-ATPase, an essential, broadly expressed transport enzyme, is well studied for its ER-to-Golgi secretory trafficking and subsequent delivery to the plasma membrane (Ackermann and Geering, 1990; Jaunin et al., 1992; Tokhtaeva et al., 2009, 2010). When we immunolabeled DGCs infected with pUEGshSTK25 at $14 \mathrm{dpi}$, we observed cell surface expression of $\mathrm{Na}, \mathrm{K}-$ ATPase, as found in control neurons (Fig. $2 G$ ). These findings showed that ER-to-Golgi and post-Golgi secretory trafficking is likely not affected upon STK25 knockdown.

Together, this set of experiments suggests that STK25 is essential for the asymmetric Golgi repositioning during early DGC integration.

Having shown that STK25 is necessary for Golgi positioning, we next tested whether STK25 is also required for dendrite establishment in adult-born DGCs. We analyzed reconstructed images of shRNA- or control adult-born DGCs at 14 dpi (Fig. $3 A, B$ ). As expected, most control neurons (75\%, Fig. $3 A, B$, top) at $14 \mathrm{dpi}$ had 2 neurites, with only a single primary dendrite pointing to the molecular layer. In contrast, most neurons infected with pUEGshSTK25 (82\%, Fig. $3 A, B$, top) exhibited $>2$ neurites that included multiple thin and few thick neurites. Quantification of the dendrite-like neurite number showed that most pUEG-shSTK25infected cells had $>4$ neurites each, compared with only 2 neurites in control (average neurite number per cell, 4.6, pUEG-shSTK25; 2.4, control; Fig. $3 B$, top left). Moreover, the average percentage of cells with $>2$ neurites was $82 \%$ in pUEG-shSTK25 DGCs compared with only $\sim 25 \%$ in control (Fig. $3 B$, top right), as confirmed by the cumulative distribution of percentage of cells with varying number of neurites in control versus pUEG-shSTK25infected neurons (Fig. 3B, bottom). To determine whether these abnormal neurites were dendrites, we immunolabeled pUEGshSTK25-infected DGCs with the dendritic marker MAP2 and found neurite labeling with MAP2 at their proximal end and along their length (Fig. 3C, arrows), indeed suggesting their dendritic identity. Strikingly, in pUEG-shSTK25 cells, we found that

\footnotetext{
(Figure legend continued.) pUEG-shSTK25 (green) into dentate gyrus of 4- to 6-week-old wildtype mice. Examination performed at $14 \mathrm{dpi}$. Bottom, Confocal images ( $60 \mu \mathrm{m} z$ stacks) of representative pUEG-shSTK25-infected DGCs (green) at $14 \mathrm{dpi}$, coimmunostained for DCX and STK25, to assess STK25 downregulation in pUEG-shSTK25-infected DGCS. Scale bar, $10 \mu \mathrm{m}$. Efficient downregulation of STK25 expression was visible in pUEG-shSTK25-infected DGCs compared with neighboring control adult-born DGCs labeled with DCX. D, Top, Paradigm for coinjection of pUEG-shSTK25 (green) together with pUX-dTomato as internal control into dentate gyrus of 4- to 6-week-old wild-type mice. Examination performed at $14 \mathrm{dpi}$. Bottom, Confocal images (60 $\mu \mathrm{m} z$ stacks) of Golgi labeling in representative pUEG-shSTK25-infected DGCs (green) at $14 \mathrm{dpi}$, immunostained for GRASP65 (gray). GCL visualized by DAPI staining. Scale bar, $20 \mu \mathrm{m}$. Images represent cells with abnormal neurites, with Golgi dispersed throughout the soma and localized at the neuritic base. Higher-magnification images (of boxed regions, top) showing Golgi localization to the abnormal neurite base (shown at bottom). Scale bar, $4 \mu \mathrm{m}$. E, 2D traces of GRASP65 fluorescence in representative pUEG-shSTK25 or control pUX-dTomato-expressing DGCs at $14 \mathrm{dpi}$, to illustrate Golgi localization. Scale bar, $10 \mu \mathrm{m}$. $\boldsymbol{F}$, Quantification of GRASP65 fluorescence in individual pUEG-shSTK25 or control pUXdTomato-expressing DGCs at $14 \mathrm{dpi}$. The soma was divided into four quadrants (apical, basal, and two laterals), right and left (apical quadrant, oriented to the ML; basal quadrant, oriented to the hilus), and average percentage of total fluorescence intensity of GRASP65 fluorescence was calculated for each quadrant (control, 11 cells; shSTK25, 9 cells; from at least 3 mice). Scale bar, $4 \mu \mathrm{m}$. G, Confocal images (60 $\mu \mathrm{m} z$ stacks) of representative pUEG-shSTK25-infected DGCs (red, right) at $14 \mathrm{dpi}$, immunostained for Na,K-ATPase (green). Neighboring adult-born DCXpositive DGCs served as control (left). Scale bar, $10 \mu \mathrm{m}$. 3D surface reconstructions were created using the mixed model rendering function (Imaris). Cell surface expression of Na,K-ATPase indicates fidelity of Golgi secretory function.
}

each neurite showed Golgi localization at its base (Fig. 2D,E), suggesting the role of the Golgi complex in the abnormal persistence of these neurites.

To exclude confounding effects of STK25 downregulation on early development of newborn DGCs, we examined effects of induced STK25 downregulation $3 \mathrm{~d}$ after neuronal birth (3 dpi). We generated a new retroviral construct for inducible shRNAdirected knockdown (Fig. 3D), pSIREN-shSTK25, in which shRNA expression and subsequent STK25 downregulation are DOX-dependent. Two shRNA sequences targeting mouse STK25 (pSIREN-shSTK25-245 and -665) efficiently downregulated the expression of targeted mouse dTom-STK 25 coexpressed in HEK293 cells upon induction with DOX, compared with cells without DOX induction, whereas control pSIREN vector had no effect (Fig. 3D). Each of the two pSIREN-shSTK25 retroviruses was injected separately into the dentate gyrus of 4- to 6-week-old mice, and STK25 downregulation was induced by DOX administration at $3 \mathrm{dpi}$ (Fig. $3 E$ ), a time by when neuronal differentiation has occurred, and which precedes neurite remodeling and primary dendrite establishment in adult-born DGCs. The dTomato control virus (pUX-dTom) was coinjected as internal control. We analyzed newborn DGCs at 14 dpi upon STK25 knockdown induced at $3 \mathrm{dpi}$ and found that most neurons exhibited multiple neurites that extended to the granule cell layer and the hilus (Fig. $3 E)$, a pattern like that observed with the constitutive pUEGshSTK25 vector (Fig. $3 A, B$ ), and unlike the single dendrite found in control neurons. Quantification of all primary neurites extending from the soma demonstrated that, upon inducible knockdown of STK25, a great majority of neurons (84\%, pSIRENshSTK25; 40\%, control) exhibited $>2$ neurites (Fig. 3F, middle), and average number of neurites in each cell increased to $\sim 4$ (Fig. $3 F$, left; 3.7, pSIREN-shSTK25; 2.5, control), confirmed by the cumulative distribution of percentage of cells with varying number of neurites in control versus pSIREN-shSTK25-infected neurons (Fig. 3F, right).

Our data support a critical role for STK25 in neurite remodeling during initial dendrite patterning of adult-born DGCs. The mispositioning of the Golgi apparatus upon STK25 downregulation, together with our findings in Figure 1 showing the potential role of the Golgi asymmetric localization in dendrite formation, suggests that STK25 might mediate dendrite establishment of adult-born DGCs by regulating Golgi repositioning.

\section{Knockdown of the STK25 cofactor STRAD impaired dendrite establishment of adult-born DGCs}

STK25 was shown to associate with and act downstream of the polarity complex assembled by the adaptor protein STRAD (Matsuki et al., 2010), a key protein complex in embryonic neuronal polarization (Barnes et al., 2007; Shelly et al., 2007), in a manner that depended on Golgi localization (Matsuki et al., 2010). Indeed, in our analysis of the single-cell transcriptome data (Fig. 2A), we found that STRAD mRNA was expressed in DCX-positive cells. Quantification of the expression of STRAD in adult-born neurons by coimmunolabeling with DCX showed that nearly all DCX-positive cells express STRAD (Fig. 4A). We therefore examined the role of STRAD in Golgi localization and dendrite establishment in adult-born DGCs. We generated retroviral mouse STRADshRNA vector, $\mathrm{pUEG}$-shSTRAD, and coinjected into the dentate gyrus of 4- to 6-week-old mice, together with dTomato control virus (pUX-dTom). We confirmed that these viruses efficiently downregulated STRAD expression in these neurons (Fig. 4B). Golgi localization was examined in infected neurons by GRASP65 immunostaining, at $14 \mathrm{dpi}$ (Fig. 4C,D). 2D traces of the Golgi in 
A
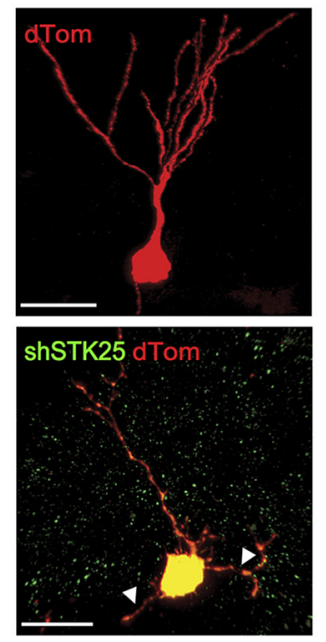

C

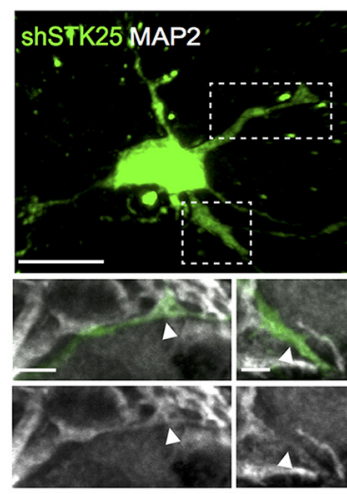

$\mathbf{F}$

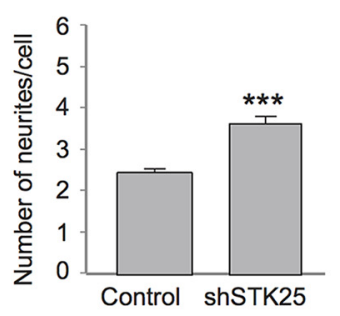

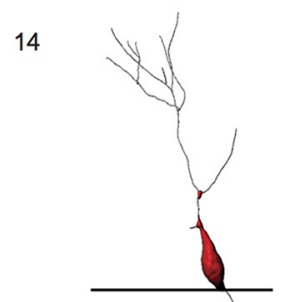

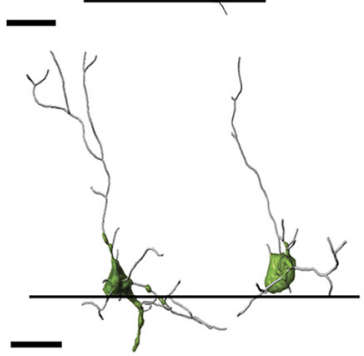

D

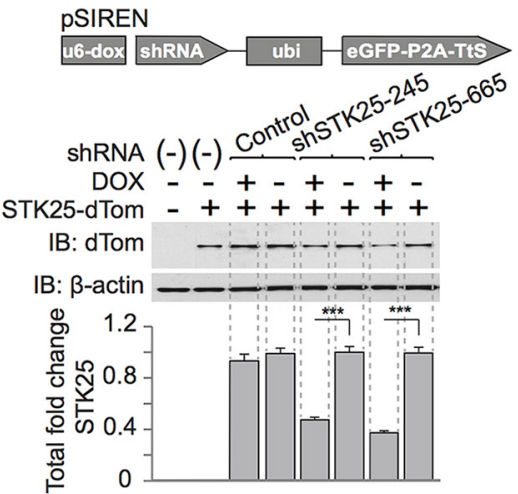

B
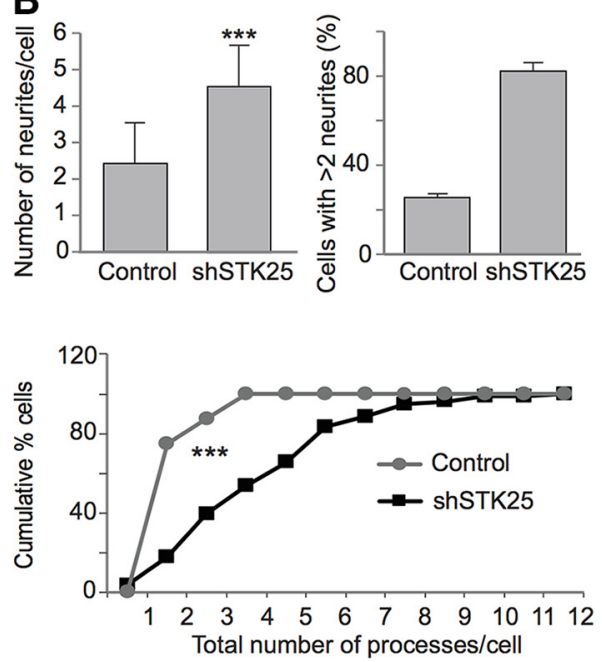

E
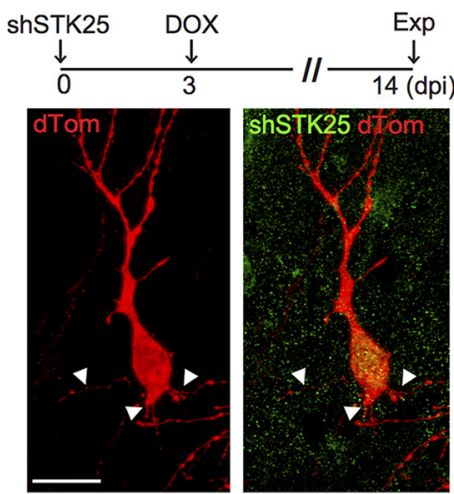
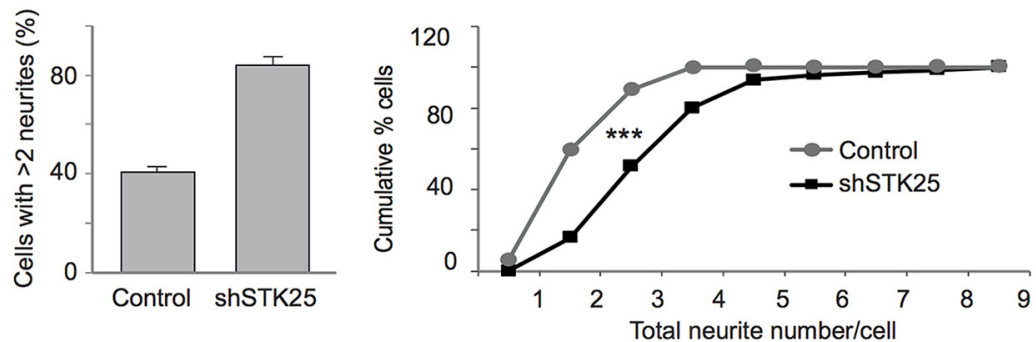

Figure 3. STK25 regulates neurite elimination during early DGC integration. $A$, Confocal images ( $60 \mu \mathrm{m} z$ stack) of representative pUEG-shSTK25 (together with pUX-dTomato) expressing DGCS, at $14 \mathrm{dpi}$ (left). Scale bar, $20 \mu \mathrm{m}$. Arrowheads indicate aberrant neurites. Right, 3D Traces of the neuritic arbor from confocal images of representative pUEG-shSTK25 or control pUX-dTomatoexpressing DGCs, at $14 \mathrm{dpi}$. Scale bar, $20 \mu \mathrm{m}$. B, Quantification of the average total number of neurites per cell (top, left) in pUEG-shSTK25 or control pUX-dTom-expressing DGCs, at $14 \mathrm{dpi}$, including all primary neurites directly extending from the soma (shSTK25, 78 cells; Control, 16 cells; from 4 separate mice; Student's two-sided $t$ test, $p=2.4232 \mathrm{E}-04, \alpha=0.05$ ); the average percentage of pUEG-shSTK25 or control pUX-dTomato-expressing DGCs with $>2$ neurites (top, right); and cumulative percentage plots of total number of neurites per cell (bottom), in pUEG-shSTK25 or control pUX-dTomato-expressing DGCs ( $p<0.05, p=1.71 \mathrm{E}-04$ Kolmogorov-Smirnov test). Same dataset was used for all quantifications in $\boldsymbol{B}$. C, Confocal image (30 $\mu \mathrm{m} z$ stack) of a representative pUEG-shSTK25-expressing DGC (green) at 14 dpi, with abnormal neurites, immunolabeled with the somatodendritic marker MAP2 (gray). Scale bar, $10 \mu \mathrm{m}$. Bottom, Higher-magnification images of the abnormal neurites (boxed regions), showing their labeling with MAP2 (arrowheads). Scale bar, $4 \mu \mathrm{m}$. D, Top, Schematic depiction of the retroviral pSIREN-ubi-GFP-P2A-tTs (pSIREN) mouse STK25-shRNA vector, pSIREN-shSTK25, for shRNA-inducible expression under the control of tet-on-U6 promoter in DOX-dependent manner, and constitutive expression of EGFP linked via the P2A sequence to the tetracycline-sensitive (Tts-S) element, under the control of ubiquitin promoter. Bottom, Immunoblots of HEK-293 whole-cell extracts cotransfected with pSIREN-STK25 shRNA constructs (shSTK25-245 or shSTK25-665) together with targeted mouse pCDNA3-dTom-STK25, treated with DOX or left untreated. "Control," cotransfection with empty pSIREN vector. dTom-STK25 levels were quantified in pSIREN-STK25 shRNA transfected cells upon DOX induction, as the fold change ( \pm SEM, $n=3, p<0.05$, from left to right, $p=3.25054 \mathrm{E}-06, p=3.87002 \mathrm{E}-06$ ) relative to control cells without DOX treatment, normalized to $\beta$-actin. E, Top, Paradigm for coinjection of pSIREN-shSTK25 (green, shSTK25-245 or shSTK25-665), together with pUX-dTomato as internal control, into dentate gyrus of 4- to 6-week-old mice, with shRNA expression induced at $3 \mathrm{dpi}$, and cell examination at $14 \mathrm{dpi}$. Bottom, Confocal images of representative DGCs coexpressing pSIREN-shSTK25 (green) together with control pUX-dTomato (red), at 14 dpi, with shRNA expression induced at 3 dpi. Arrowheads indicate aberrant neurites. Scale bar, $20 \mu \mathrm{m}$. $\boldsymbol{F}, 0$ uantification of the average total number of neurites per cell (left) (shSTK25, 80 cells; Control, 37 cells; from 4 separate mice, with 2 additional mice without D0X treatment; Student's two-sided ttest, $p=9.38 \mathrm{E}-07$, $\alpha=0.05$ ), average percentage of DGCs with $>2$ neurites per cell (middle), and cumulative percentage plots for total number of neurites per cell (right) $(p<0.05, p=4.63 \mathrm{E}-05 \mathrm{Kolmogorov}-$ Smirnov test), for pSIREN-shSTK25 or control pUX-dTomato-expressing DGCs, at 14 dpi, with shRNA expression induced at 3 dpi. Analysis included all primary neurites directly extending from the soma. Same dataset was used for all analyses in $F .^{* * *} p \leq 0.001$. 
A

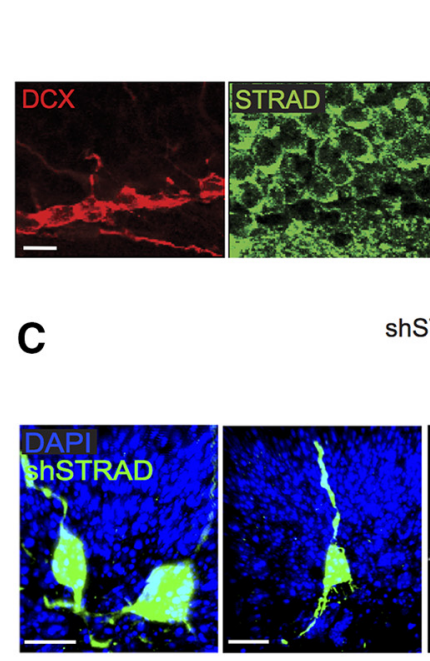

E
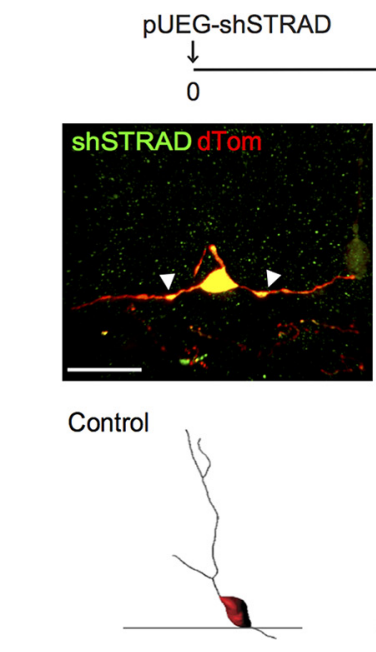

G

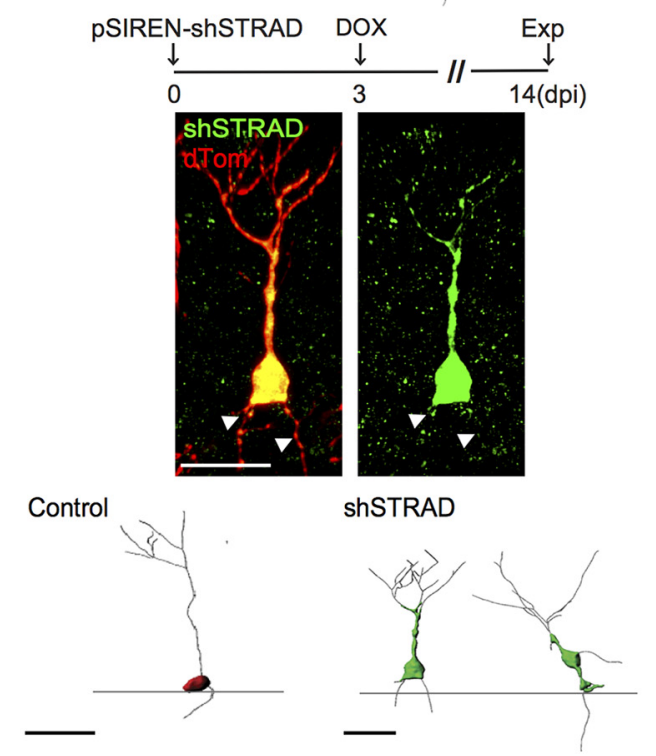

Exp

$14(\mathrm{dpi})$
B pUEG : u6-shSTRAD-EF1 $\alpha-$ GFP
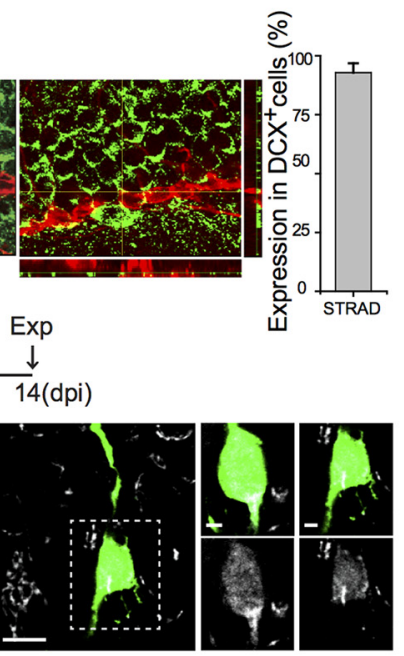

$\mathbf{F}$
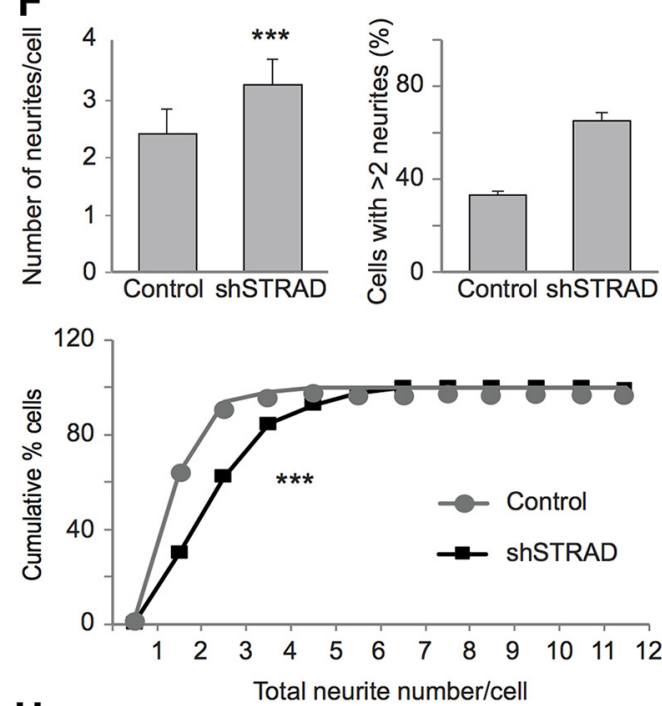

н
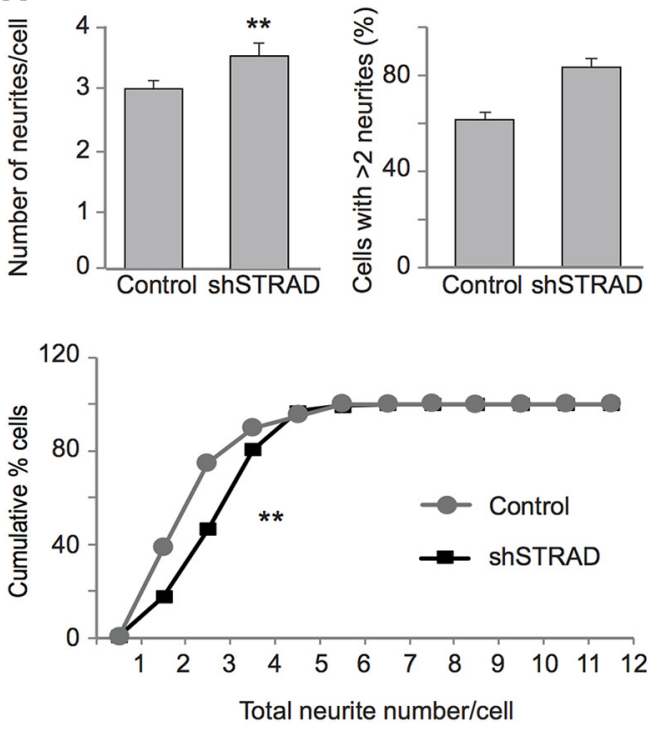

Figure 4. STRAD mediates both Golgi positioning and neurite elimination during early DGC integration. $\boldsymbol{A}$, Left, Confocal images of representative adult-born DGCs from wild-type 4- to 6-week-old mouse hippocampus immunolabeled with DCX together with antibody for STRAD. Scale bar, $10 \mu$ m. Coexpression was confirmed by 3-plane analysis. Right, Quantification of the average percentage of DCX-positive adult-born DGCs expressing STRAD determined based on coimmunolabeling with DCX (STRAD, 60 cells; from 4 separate mice). These examinations showed that nearly all DCX-positive cells express STRAD. B, Top, Schematic depiction of retroviral mouse STRAD-shRNA vector, pUEG-shSTRAD, and paradigm for injection of pUEG-shSTRAD (green) into dentate gyrus of 4- to 6-week-old mice, and cell examination at $14 \mathrm{dpi}$. Bottom, Confocal images (60 $\mu \mathrm{m} \mathrm{z}$ stacks) of representative pUEG-shSTRAD-infected (Figure legend continues.) 
individual pUEG-shSTRAD-infected cells (Fig. 4D) showed that, upon STRAD downregulation, the Golgi was highly dispersed throughout the soma, similar to that observed with STK25 downregulation (Fig. $2 D, E$ ), and in contrast to the compact Golgi localization to the primary dendrite in control DGCs. Importantly, the Golgi was localized to the base of multiple abnormal neurites observed upon STRAD knockdown (Fig. 4C-E), as that with STK25 knockdown (Fig. 2D,E). These findings demonstrate that both STK25 and STRAD are required for Golgi positioning during early DGC integration.

We next tested the requirement of STRAD in dendrite establishment in adult-born DGCs, upon its knockdown in adult stem cells with pUEG-shSTRAD (Fig. 4E,F), or its knockdown induced in differentiated DGCs at 3 dpi with pSIREN-shSTRAD (Fig. 4G,H). We examined and quantified neurite number in infected neurons at $14 \mathrm{dpi}$ and found that the majority of neurons upon STRAD downregulation exhibited multiple thin and thick primary neurites extending to the GCL and the hilus (Fig. 4E, G), unlike control morphology. Quantification of average total neurite number (Fig. 4F, $H$, top left) revealed a significant increase in each cell upon STRAD downregulation compared with control (3.3, pUEG-shSTRAD; 3.5, pSIREN-shSTRAD), and an increase in the percentage of cells demonstrating $>2$ neurites (Fig. $4 F, H$, top right; 70\%, pUEG-shSTRAD, 36\%, control; 82\%, pSIRENshSTRAD, $61 \%$ control). This was further confirmed by the cumulative distribution of percentage of cells with varying number of neurites in pUEG-shSTRAD (Fig. $4 F$, bottom) or pSIRENshSTRAD-infected neurons (Fig. $4 H$, bottom), compared with control. Together, our findings show that STK25 and STRAD are both critical regulators of Golgi localization as well as the initial dendrite establishment in adult-born DGCs.

How do STK25 and STRAD exert their regulation on Golgi complex localization? We performed coimmunoprecipitation experiments in HEK-293 cells and observed that the association

\footnotetext{
$\leftarrow$

(Figure legend continued.) DGCs (green) at $14 \mathrm{dpi}$, coimmunostained for DCX and STRAD, to assess STRAD downregulation in pUEG-shSTRAD-infected DGCs. Scale bar, $10 \mu \mathrm{m}$. Efficient downregulation of STRAD expression was visible in pUEG-shSTRAD-infected DGCs compared with neighboring control adult-born DGCs labeled with DCX. C, Top, Paradigm for coinjection of pUEG-shSTRAD (green) together with pUX-dTomato as internal control, into dentate gyrus of 4to 6-week-old mice, and cell examination at $14 \mathrm{dpi}$. Bottom, Confocal images (60 $\mu \mathrm{m} z$ stacks) of Golgi labeling in representative pUEG-shSTRAD-infected DGCs (green) at 14 dpi, immunostained for GRASP65 (gray). GCL visualized by DAPI staining. Scale bar, $20 \mu \mathrm{m}$. Images represent cells with abnormal neurites, with Golgi dispersed throughout the soma and localized at the neuritic base. Higher-magnification images (of boxed regions) showing Golgi localization to the abnormal neurite base, presented on the right. Scale bar, $4 \mu \mathrm{m} . D, 2 D$ traces of GRASP65 fluorescence in representative pUEG-shSTRAD expressing DGCs at 14 dpi to illustrate Golgi localization. Scale bar, $10 \mu \mathrm{m}$. $\boldsymbol{E}, \boldsymbol{G}$, Top, Paradigm for injection of pUEG-ShSTRAD (E) or pSIRENshSTRAD $(G)$ (green), together with pUX-dTomato (red) as internal control, into dentate gyrus of 4- to 6-week-old mice, with the pSIREN-shSTRAD expression induced at $3 \mathrm{dpi}(\boldsymbol{G})$, and cell examination performed at $14 \mathrm{dpi}$. Bottom, Confocal images and $3 \mathrm{D}$ traces of the neuritic arbor of representative DGCs expressing pUEG-shSTRAD (E) or pSIREN-shSTRAD ( $\boldsymbol{G}$ ) (green) together with control pUX-dTomato (red) at 14 dpi. Arrowheads indicate aberrant neurites. Scale bar, 20 $\mu \mathrm{m} . \boldsymbol{F}, \boldsymbol{H}$, Quantification of the average total number of neurites per cell (top, left), the average percentage of DGCs with $>2$ neurites per cell (top, right), and cumulative percentage plots for total number of neurites per cell (bottom) for pUEG-ShSTRAD $(\boldsymbol{F})$ or pSIREN-shSTRAD $(\boldsymbol{H})$, and control pUX-dTomato-expressing DGCs, at $14 \mathrm{dpi}$, with pSIREN-shSTRAD expression induced at $3 \mathrm{dpi}(\boldsymbol{H})$. For number of neurites, pUEG-shSTRAD, 162 cells; control, 50 cells; from 4 separate mice; Student's two-sided $t$ test, $p=9.38 \mathrm{E}-07, \alpha=0.05$; pSIREN-shSTRAD, 90 cells; Control, 60 cells; from 4 separate mice, with 2 additional mice without DOX treatment; Student's twosided $t$ test, $p=1.10 \mathrm{E}-03, \alpha=0.05$. For cumulative percentage plots, $p<0.05, p=2.24 \mathrm{E}-04$ (pUEG-shSTRAD), $p=0.0019$ (pSiren-shSTRAD) Kolmogorov-Smirnov test. Analysis included all primary neurites directly extending from the soma. Same dataset was used for all quantifications. ${ }^{* *} p \leq 0.01,{ }^{* *} p \leq 0.001$.
}

between STK25 and STRAD resulted in significant stabilization of the STK25 protein (Fig. 5A). Further analysis of the single-cell transcriptome data showed that the Golgi matrix protein GM130, a Golgi signaling-scaffold that enables asymmetric subcellular Golgi positioning (Matsuki et al., 2010; Huang et al., 2014), was also expressed in newborn DGCs (Fig. 2A). This finding was important because, during embryonic neuronal polarization, STK25 was shown to regulate Golgi localization through the recruitment of GM130 (Preisinger et al., 2004; Matsuki et al., 2010). It is thus possible that STK25-GM130 mediate Golgi localization in adultborn DGCs via association with the STRAD polarity complex. Structural studies showed cooperative interactions among the STRAD polarity complex members, which include the kinase LKB1 and the adaptor protein MO25 (Boudeau et al., 2004; Zeqiraj et al., 2009). Therefore, the association of STK25 with STRAD might stabilize a Golgi signaling complex via interaction with GM130, necessary for Golgi-polarized positioning in newborn DGCs. In support, we found that GM130 associated with STRAD (Fig. 5G) and that coexpression of GM130 with either STRAD or STK25 in HEK-293 cells resulted in significant stabilization of the GM130 protein (Fig. 5B). In support of these biochemical expression studies, our coimmunolabeling experiments of DCX-positive adult-born DGCs in 4- to 6-week-old mouse hippocampus showed that STK25 was predominantly found in distinct somatic structures at the base of the apical dendrite (Fig. 5C,D) and that STK25 colocalized with STRAD (Fig. 5C). Furthermore, STK25 was found to colocalize with the Golgi marker GRASP65 (Fig. 5D). Together, these biochemical and immunolabeling findings showed that STK25 and STRAD form and stabilize a Golgi signaling complex with GM130 and that the complex is localized to the Golgi.

Our biochemical and immunohistochemical examinations together with the knockdown studies in adult-born DGCs showed that STK25 and STRAD association might mediate Golgi positioning via recruitment and stabilization of the Golgi matrix protein GM130. The STK25-STRAD-GM130 Golgi complex might regulate both Golgi positioning and the initial dendritic establishment in adult-born DGCs.

\section{A STRAD mutation underlying the neurodevelopmental disorder polyhydramnios, megalencephaly and symptomatic epilepsy (PMSE) destabilizes GM130 and disrupts Golgi positioning and initial dendrite patterning of adult-born DGCs}

STRAD is part of a polarity complex composed of the serine/ threonine kinase LKB1 and the adaptor protein MO25 (Boudeau et al., 2004; Milburn et al., 2004). The stabilization of GM130 and STK25 upon association with STRAD (Fig. $5 A, B$ ) suggests that the interaction of STK25 with STRAD/MO25/LKB1 may constitute a complex necessary for asymmetric Golgi localization. In support, we found that, upon coexpression with STRAD, LKB1, or MO25, there was a substantial stabilization of the STK25 protein (data not shown). A homozygous, partial deletion in the STRAD $\alpha$ gene, which generates a 180 C-terminal amino acid truncation of the STRAD protein (Fig. 5E), underlies the human neurodevelopmental disorder PMSE, characterized by epilepsy and cognitive and motor disability (Puffenberger et al., 2007; Orlova et al., 2010). The C-terminal 180 amino acid region of STRAD truncated in PMSE is highly conserved among the mouse, rat, and human STRAD $\alpha$ proteins, which in structural studies (Zeqiraj et al., 2009) was shown to be important for LKB1/STRAD/MO25 complex formation. 
A

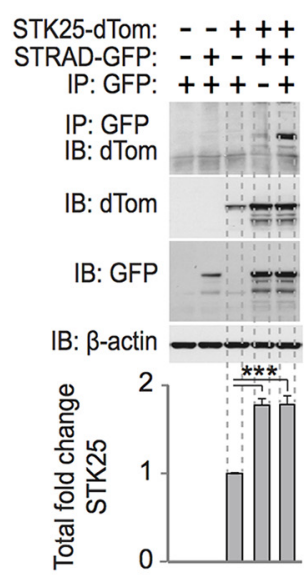

$\mathbf{F}$

MO25-dTom: -+++

LKB1-dTom: - - - - -

STRAD-GFP: $\overline{\mathbf{t}}+\overline{+}+$

A180-STRAD-GFP: $\mathbf{-}-\mathbf{+}$

IP: GFP: +++++

IP: GFP

IB: dTom

IB: dTom

IB: GFP
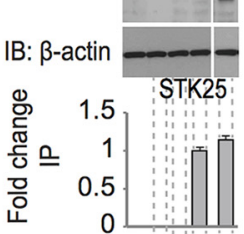

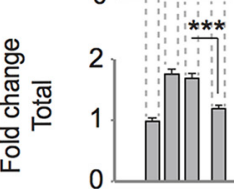

B

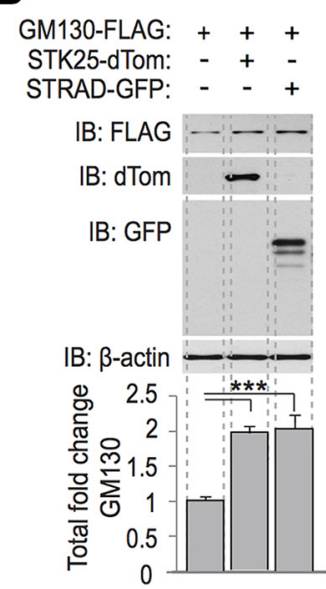

C
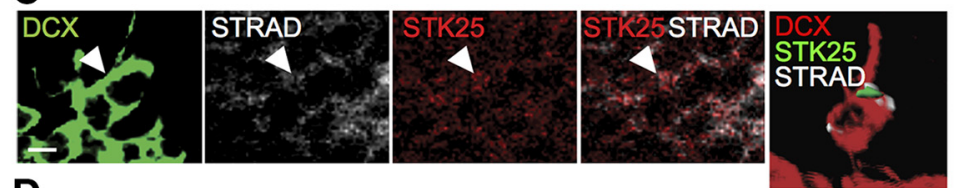

D

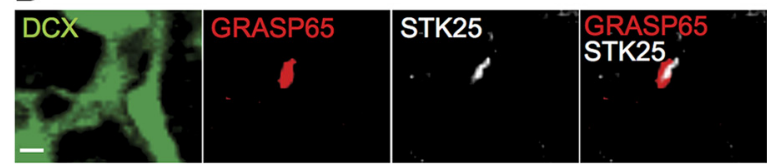

E

Wild-type

STRAD

431 aa

$\triangle 180$

STRAD-PMSE 251 aa

G

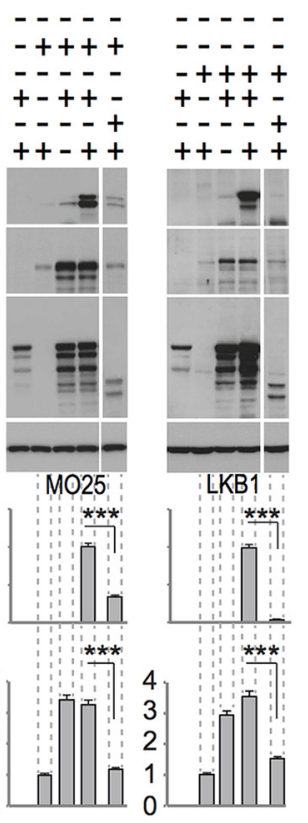

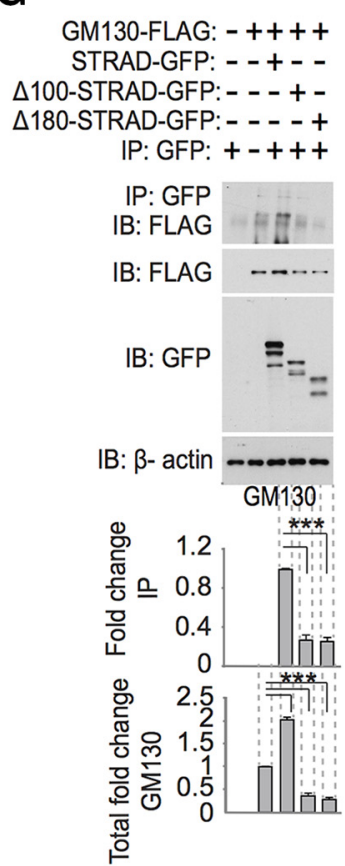

H

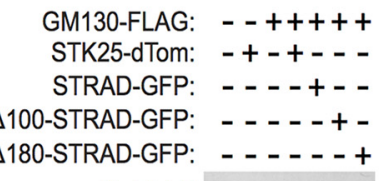

A180-STRAD-GFP: - - - - + +

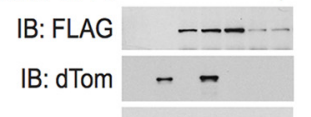

IB: GFP

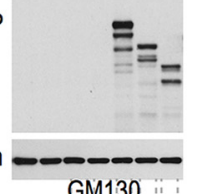

IB: $\beta$ - actin

GM130

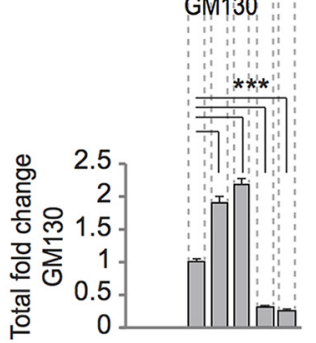

Figure 5. STK25-STRAD complex formation and localization to the Golgi are disrupted upon expression of STRAD-PMSE- $\Delta 180$. $A$, Coimmunoprecipitation of STK25 with STRAD, from HEK-293 cell lysates coexpressing dTom-STK25 together with GFP-STRAD. Whole-cell extracts were subjected to immunoprecipitation (IP) for GFP and immunoblotting (IB) for dTom. Coimmunoprecipitation with normal serum served as control. Total cell lysates were also immunoblotted for dTom or EGFP to test dTom-STK25 or GFP-STRAD expression, and for $\beta$-actin to control for protein loading. dTom-STK25 total protein levels were quantified upon coexpression with GFP-STRAD, as fold increase ( \pm SEM, $n=3, p<0.05, p=0.000434272$ ) relative to control cells in which dTom-STK25 was expressed alone, normalized to $\beta$-actin. Association between STK25 and STRAD resulted in STK25 stabilization. B, Immunoblotting of HEK-293 whole-cell lysates coexpressing Flag-GM130 together with either dTom-STK25 or GFP-STRAD, for Flag, dTom, or GFP. Flag-GM130 protein levels were quantified upon coexpression with dTom-STK25 or GFP-STRAD as the fold increase $( \pm$ SEM, $n=3$, $p<0.05, p=0.000626417$, Stk25; $p=8.19784 \mathrm{E}-06$, STRAD), relative to control cells in which Flag-GM130 was expressed alone, normalized to $\beta$-actin. Coexpression of GM130 with either STK25 or STRAD resulted in GM130 stabilization. C, D, Confocal images (60 $\mu \mathrm{m} z$ stacks) of representative adult-born DGCs from wild-type 4- to 6-week-old mouse hippocampus coimmunolabeled with DCX together with antibodies for STK25 and STRAD (C), or STK25 and GRASP65 (D). Scale bar, $10 \mu \mathrm{m}$. STK25 colocalized with STRAD (C), as well as with GRASP65 (D), indicating localization to the Golgi. Right, Higher-magnification image of 3D reconstructions of each channel (mixed model rendering function, Imaris), showing colocalization between STK25 (green) and STRAD (gray) at the base of the apical dendrite, in a DCX-positive (red) adult-born DGC (C). Scale bar, $10 \mu \mathrm{m}$. E, Schematic depiction of WT or the 180 C-terminal amino acid truncation of STRAD, which underlies the human neurodevelopmental disorder PMSE. $\boldsymbol{F}$, G, Coimmunoprecipitation of $\Delta$ 180-STRAD compared with WT-STRAD, with STK25, M025, and LKB1 (F), or with GM130 (G), from HEK-293 cell lysates coexpressing GFP- $\triangle 180$-STRAD or GFP-WT-STRAD, together with dTom-STK25, dTom-M025, and dTom-LKB1 (F), or Flag-GM130 (G). A mutant STRAD with 100 amino acid C-terminal truncation, GFP- $\Delta$ 100-STRAD, was also examined (G). Whole-cell extracts were subjected to IP to GFP $(\boldsymbol{F}, \boldsymbol{G})$, and IB for dTom $(\boldsymbol{F})$ or Flag $(\boldsymbol{G})$. Coimmunoprecipitation with normal serum served as control. Total cell-lysates were immunoblotted for Flag, dTom, or GFP. Flag-GM130 (G) or dTom-STK25, dTom-M025, or dTom-LKB1 (F) levels were quantified upon their coexpression with GFP- $\Delta 180$-STRAD or GFP-WT-STRAD, in the coimmunoprecipitation or total cell lysates, as a measure of their association with STRAD proteins (top) or their stabilization (bottom), respectively, as the fold increase ( \pm SEM, $n=3-5, p<0.05$ ) relative to respective control, normalized to $\beta$-actin. These experiments showed that $\triangle 180$-STRAD associated with STK25 but did not stabilize the STK25 protein, whereas $\triangle 180$-STRAD lost association with GM130, M025, or LKB1, compared with WT-STRAD. $\boldsymbol{H}$, Immunoblotting of HEK-293 whole-cell lysates coexpressing Flag-GM130 together with GFP- $\Delta 180$-STRAD or GFP-WT-STRAD, for Flag or GFP. dTom-STK25 and GFP- $\Delta 100$-STRAD were also included in the analysis. Flag-GM130 level was quantified upon coexpression with $\Delta 180$-STRAD compared with coexpression with WT-STRAD or STK25, as the fold change ( \pm SEM, $n=3, p<0.05, p=8.23507 \mathrm{E}-05$, WT-STRAD; $p=3.49943 \mathrm{E}-05$, STK25; $p=5.76896 \mathrm{E}-05, \Delta 100-\mathrm{STRAD} ; p=$ $1.81666 \mathrm{E}-06, \Delta 180$-STRAD) relative to control cells in which Flag-GM130 was expressed alone, normalized to $\beta$-actin. Coexpression of GM130 with either WT-STRAD or STK25 caused its stabilization, whereas its coexpression with $\Delta 180-$ STRAD or $\Delta 100-$ STRAD caused significant decrease in GM130 level (see also $B$ ). ${ }^{* * *} p \leq 0.001$. 
A pTet-STRAD-PMSE- $\Delta 180$
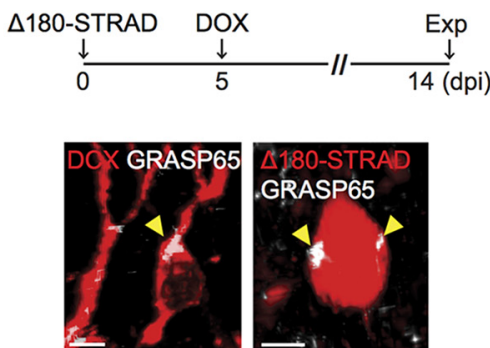

D
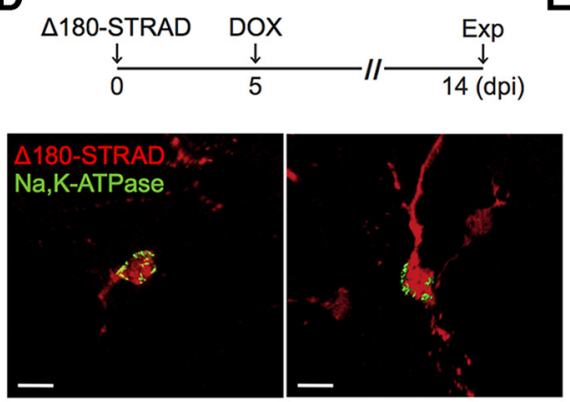

F
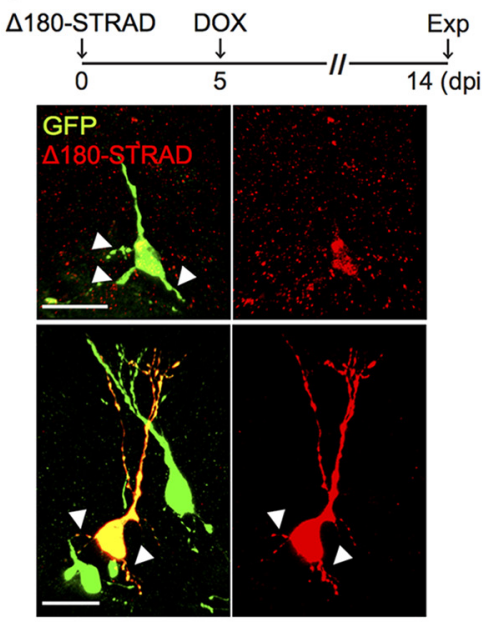

H

pUEG : u6-shGM130-EF1 $\alpha-$ GFP shGM130

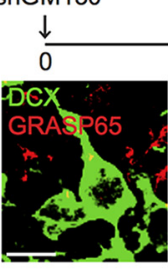

I

Control | shGM130 GRASP65

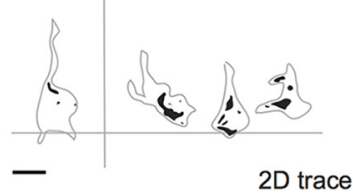

E
B

C
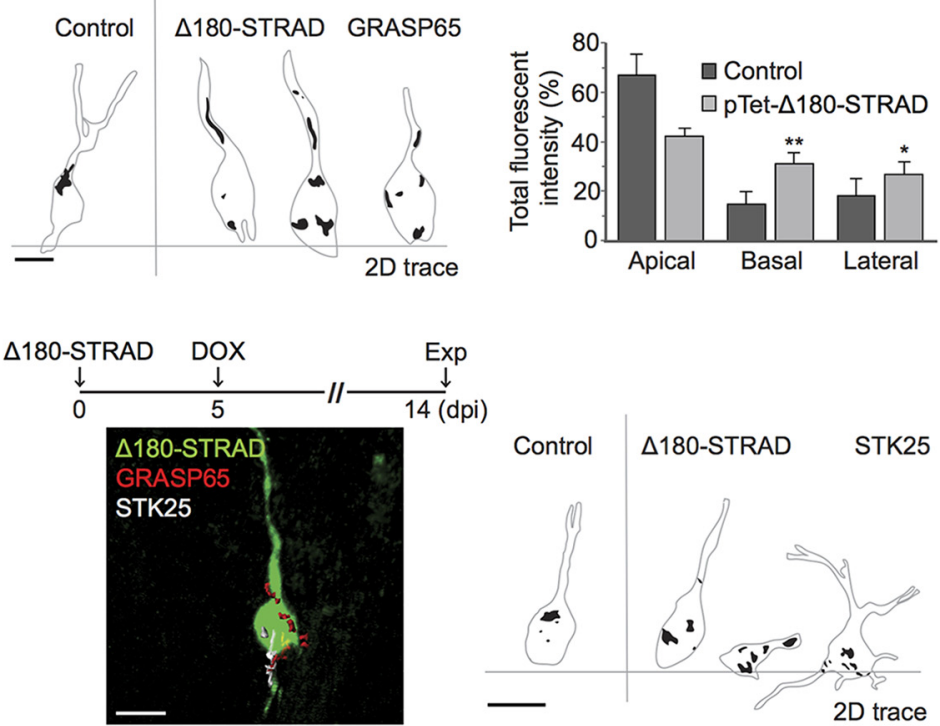

G
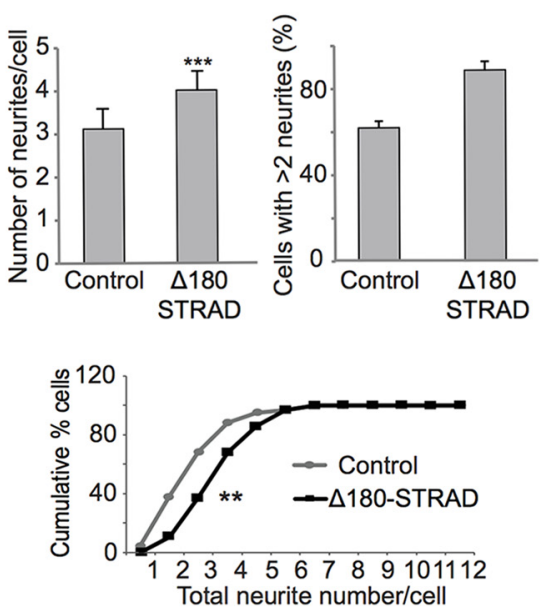

K
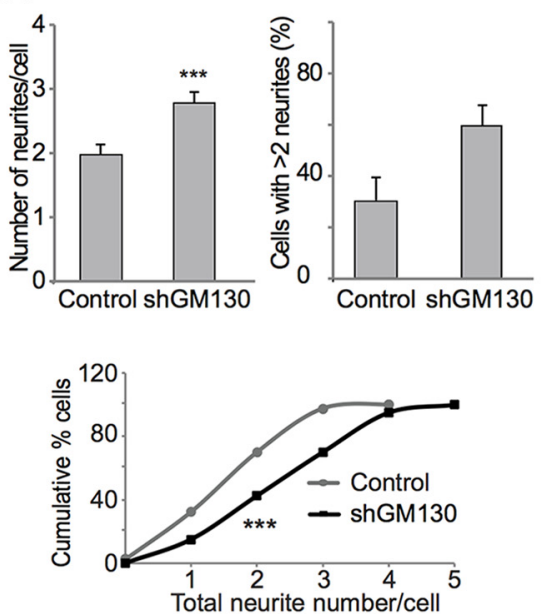

Figure 6. Golgi positioning and neurite elimination are disrupted upon expression of STRAD-PMSE- $\Delta 180$. $\boldsymbol{A}$, Top, Schematic depiction of D0X-inducible retroviral construct for mouse STRAD-PMSE- $\Delta 180$ fused to dTom or GFP (pTet-STRAD-PMSE- $\Delta 180$ ), and paradigm for injection of pTet-STRAD-PMSE- $\Delta 180$ into dentate gyrus of 4- to 6-week-old mice, with STRAD-PMSE- $\Delta 180$ expression induced at $5 \mathrm{dpi}$, and cell examination at $14 \mathrm{dpi}$. Bottom, Confocal images (60 $\mu \mathrm{m} z$ stacks) of Golgi labeling in two representative (Figure legend continues.) 
We generated the PMSE mutant form of mouse STRAD, with a truncation of the C-terminal 180 amino acids, STRAD-PMSE$\Delta 180$ (Fig. $5 E$ ), and determined its association with STK25 or GM130, as well as with MO25 or LKB1, by coimmunoprecipitation upon coexpression in HEK-293 cells. These experiments resulted in two crucial observations. First, the STRAD-PMSE- $\Delta 180$ maintained association with STK25, but not with MO25 or LKB1 (Fig. 5F). Furthermore, although STRAD-PMSE- $\Delta 180$ interacted with STK25, this association did not result in STK25 protein stabilization in contrast to STK25 stabilization upon its association with wild-type STRAD (Fig. 5F). The lack of STK25 stabilization upon association with STRAD-PMSE- $\Delta 180$ likely

$\leftarrow$

(Figure legend continued.) pTet-STRAD-PMSE- $\Delta 180$-infected DGCs at 14 dpi, immunostained for GRASP65 (gray). Scale bar, $10 \mu \mathrm{m}$. Images represent STRAD-PMSE- $\Delta 180$-infected DGCS with Golgi dispersed in the soma (arrowheads) and localized at the neuritic base. Adult-born DCX-positive DGCs served as control (left). B, 2D traces of GRASP65 fluorescence in representative pTet-STRAD-PMSE- $\Delta 180$-expressing DGCs at $14 \mathrm{dpi}$, to illustrate Golgi dispersion upon STRAD-PMSE- $\Delta 180$ expression. Scale bar, $10 \mu \mathrm{m}$. C, Quantification of GRASP65 fluorescence in each of four quadrants (apical, basal, and right and left laterals) of individual STRAD-PMSE$\Delta 180$ or control DCX-positive adult-born DGCs at 14 dpi, presented as average percentage of total fluorescence intensity of GRASP65 fluorescence in each quadrant (control, 12 cells; STRADPMSE- $\Delta 180,13$ cells; from 3 mice; Student's two-sided $t$ test $p=0.1438$, apical; $p=$ 0.003434 , basal; $p=0.012295$, lateral; $\alpha=0.05$ ). $\boldsymbol{D}$, Confocal 3D surface reconstruction images ( $60 \mu \mathrm{m} z$ stacks) of two representative pTet-STRAD-PMSE- $\Delta 180$-infected DGCs (red) at $14 \mathrm{dpi}$, immunostained for $\mathrm{Na}$, K-ATPase. Scale bar, $10 \mu \mathrm{m}$. Cell surface expression of $\mathrm{Na}, \mathrm{K}-$ ATPase indicates fidelity of Golgi secretory function. $\boldsymbol{E}$, Top, Paradigm for injection of pTet-STRAD-PMSE- $\Delta 180$ (green) into dentate gyrus of 4- to 6-week-old mice, with STRADPMSE- $\Delta 180$ expression induced at $5 \mathrm{dpi}$, and cell examination at $14 \mathrm{dpi}$. Left, Highmagnification 3D reconstruction image $(60 \mu \mathrm{m} z$ stacks) of a representative adult-born DGC-expressing pTet-STRAD-PMSE- $\Delta 180$ (green), at 14 dpi, coimmunolabeled for STK25 and GRASP65, showing loss of colocalization between STK25 and GRASP65, and both their somatic dispersions. Scale bar, $10 \mu \mathrm{m}$. Right, $2 \mathrm{D}$ traces of STK25 fluorescence in representative pTetSTRAD-PMSE- $\Delta 180$ or control pUX-dTomato-expressing DGCS at 14 dpi, to illustrate STK25 somatic dispersion upon pTet-STRAD-PMSE- $\Delta 180$ expression. Scale bar, $10 \mu \mathrm{m}$. $\boldsymbol{F}$, Top, Paradigm for coinjection of pTet-STRAD-PMSE- $\Delta 180$ (red) together with pUX-EGFP as internal control, into dentate gyrus of 4- to 6-week-old mice, with STRAD-PMSE- $\Delta 180$ expression induced at $5 \mathrm{dpi}$, and cell examination at $14 \mathrm{dpi}$. Bottom, Confocal images (left) and 3D traces (right) of the neuritic arbor of representative DGCs coexpressing pTet-STRAD-PMSE- $\Delta 180$ (red) together with pUX-GFP (green) as internal control, at $14 \mathrm{dpi}$. Arrowheads indicate aberrant neurites. Scale bar, $20 \mu \mathrm{m}$. G, Quantification of the average total number of neurites per cell: STRADPMSE- $\Delta$ 180, 94 cells; Control, 60 cells; from 5 separate mice; Student's two-sided $t$ test, $p=$ $2.20 \mathrm{E}-05, \alpha=0.05$ (top left), average percentage of DGCs with $>2$ neurites per cell (top right), and cumulative percentage plots for total number of neurites per cell (bottom) $(p<0.05, p=$ 0.0012 , Kolmogorov-Smirnov test) for pTet-STRAD-PMSE- $\Delta 180$ or control pUX-EGFP expressing DGCS, at 14 dpi, with pTet-STRAD-PMSE- $\Delta 180$ expression induced at 5 dpi. Analysis included all primary neurites directly extending from the soma. Same dataset was used for all analyses in $\boldsymbol{G}$. $\boldsymbol{H}$, Top, Schematic depiction of retroviral mouse GM130-shRNA vector, pUEGshSTRAD, and paradigm for injection of pUEG-shGM130 (green) into dentate gyrus of 4- to 6-week-old mice, and cell examination at $14 \mathrm{dpi}$. Bottom, Confocal images ( $60 \mu \mathrm{m} z$ stacks) of Golgi labeling in representative pUEG-shGM130-infected DGCs (green) at 14 dpi, immunostained for GRASP65 (red). DCX-positive adult-born DGCs served as control. Scale bar, $10 \mu \mathrm{m}$. Images represent Golgi somatic dispersion upon GM130 knockdown. I, 2D traces of GRASP65 fluorescence in representative pUEG-shGM130-expressing DGCs at 14 dpi to illustrate Golgi abnormal somatic localization. Scale bar, $10 \mu \mathrm{m}$. J, Top, Paradigm for injection of pUEGshGM130 (green), together with pUX-dTomato as internal control, into dentate gyrus of 4- to 6-week-old mice, and cell examination at $14 \mathrm{dpi}$. Bottom, Confocal images and $3 \mathrm{D}$ traces of the neuritic arbor of representative DGCs expressing pUEG-shGM130 (green) or control pUX$\mathrm{dTomato}$ at $14 \mathrm{dpi}$. Arrowheads indicate aberrant neurites. Scale bar, $10 \mu \mathrm{m} . \boldsymbol{K}$, Quantification of the average total number of neurites per cell (top left), average percentage of DGCs with $>2$ neurites per cell (top right), and cumulative percentage plots for total number of neurites per cell (bottom) for pUEG-shGM130, and control pUX-dTomato-expressing DGCs, at 14 dpi. For number of neurites, pUEG-shGM130, 40 cells; control, 40 cells; from 3 separate mice; Student's two-sided $t$ test, $p=9.38 \mathrm{E}-07, \alpha=0.05$. For cumulative percentage plots, $p<0.001$, Kolmogorov-Smirnovtest. Analysis induded all primaryneurites directly extending from the soma. Same dataset was used for all analyses in $K^{*} p \leq 0.05,{ }^{* *} p \leq 0.01,{ }^{* * *} p \leq 0.001$. reflects defective complex formation with MO25/LKB1 (Zeqiraj et al., 2009).

What is the consequence of these impaired interactions on the association of GM130 with the STK25-STRAD complex and the resulting stabilization of GM130? Furthermore, what is the consequence of these impaired interactions on Golgi positioning? To answer these questions, first we examined the association of STRAD-PMSE- $\Delta 180$ with GM130 and found that they do not interact (Fig. $5 G$ ). Thus, the STRAD-PMSE- $\Delta 180$ maintained association only with STK25 but not with any other member of the Golgi signaling complex, including GM130. We therefore predicted that STRAD PMSE- $\Delta 180$ might act as a dominant-negative form, which would interfere with GM130-STK25-STRAD-MO25LKB1 Golgi complex formation. This might lead to STK25-STRAD complex disassembly, GM130 destabilization, and Golgi dispersion. To test this assumption, we quantified GM130 protein level upon coexpression with STRAD-PMSE- $\Delta 180$ and found a considerable decrease in GM130 level, compared with its expression alone or coexpression with WT-STRAD or STK25 (Fig. 5H). Together, these findings showed that expression of STRAD-PMSE- $\Delta 180$ may exert a dominant-negative effect on GM130-STK25-STRAD Golgi complex formation, resulting in GM130 destabilization.

The possible dissociation of the STK25 Golgi-associated complex (Fig. 5) and the destabilization of GM130 (Fig. 5H) upon expression of STRAD-PMSE- $\Delta 180$, as well as the Golgi somatic dispersion upon STRAD downregulation (Fig. 4C,D), leads us to predict that expression of STRAD-PMSE- $\Delta 180$ in developing DGCs might result in Golgi dispersion, associated with defects in DGC dendrite establishment. We generated a DOX-inducible retroviral construct (Kumamoto et al., 2012) for mouse STRADPMSE- $\Delta 180$ fused to dTomato or GFP (pTet-STRAD-PMSE$\Delta 180$; Fig. $6 A$ ). First, we examined effects on Golgi positioning in pTet-STRAD-PMSE- $\Delta 180$-infected DGCs at 14 dpi by GRASP65 immunostaining (Fig. $6 A, B$ ) upon induction of STRAD-PMSE$\Delta 180$ expression at $5 \mathrm{dpi}$. Induction at $5 \mathrm{dpi}$ was chosen because earlier induction of STRAD-PMSE- $\Delta 180$ expression likely resulted in considerable cell death (data not shown). Examination of Golgi localization in individual STRAD-PMSE- $\Delta 180$-infected cells (Fig. $6 A-C$ ) showed that the Golgi was dispersed throughout the soma, similar to that observed with STK25 (Fig. 2D,E) or STRAD downregulation (Fig. $4 C, D$ ), and in contrast to the compact Golgi localization to the primary dendrite in control DGCs. Importantly, the Golgi was localized to the base of multiple abnormal neurites observed upon STRAD-PMSE- $\Delta 180$ overexpression (Fig. $6 A, B$ ), as that with STK25 or STRAD downregulation. When we immunostained adult-born DGCs infected with STRAD-PMSE$\Delta 180$ with antibody to $\mathrm{Na}, \mathrm{K}-\mathrm{ATP}$ ase at $14 \mathrm{dpi}$, we found that the transporter was expressed at the cell surface (Fig. 6D), as in control neurons, indicating that Golgi secretory trafficking is likely not affected upon STRAD-PMSE- $\Delta 180$ overexpression. These findings showed that the STRAD mutation, which underlies the PMSE disorder interferes with Golgi positioning during early DGC integration.

Based on our biochemical analyses (Fig. $5 F-H$ ), we predicted that STRAD-PMSE- $\Delta 180$ would compete in a dominant-negative manner with endogenous STRAD for association with STK25 as part of the STK25-STRAD-GM130 complex, resulting in complex disassembly and its dissociation from the Golgi, with subsequent Golgi dispersion. To test this prediction, we examined the subcellular localization of STK25 in adult-born DGCs expressing STRAD-PMSE- $\Delta 180$, by immunolabeling STRAD-PMSE- $\Delta 180$ infected DGCs for STK25 at $14 \mathrm{dpi}$. These analyses showed that, unlike the restricted STK25 signal in control neurons, which was 
mostly localized to the base of the apical dendrite and colocalized with GRASP65 (Fig. 5D), STK25 was dispersed in the soma of STRAD-PMSE- $\Delta 180$-infected DGCs, it indeed showed partial localization with STRAD-PMSE- $\Delta 180$, and it was no longer associated with GRASP65 (Fig. 6E), indicating its dissociation from the Golgi. These findings support the idea that expression of STRAD PMSE- $\Delta 180$ results in the dissociation of the STK25Golgi-associated complex.

Next, we examined effects on neurite remodeling and dendrite establishment in DGCs at $14 \mathrm{dpi}$, upon induction of STRAD-PMSE- $\Delta 180$ expression at $5 \mathrm{dpi}$. We analyzed the pTetSTRAD-PMSE- $\Delta 180$-infected cells and found severe defects in dendrite establishment (Fig. $6 F, G$ ), consistent with that observed with STK25 (Fig. 3) or STRAD (Fig. 4) downregulation. Qualitative (Fig. $6 F$ ) and quantitative (Fig. $6 G$ ) examination of pTetSTRAD-PMSE- $\Delta 180$-infected cells revealed severe defects in neurite elimination and formation of the single primary dendrite, with significantly higher average total number of neurites per cell upon pTet-STRAD-PMSE- $\Delta 180$ overexpression, compared with control (Fig. $6 G$, top left). The percentage of cells with $>2$ neurites was also higher upon pTet-STRAD-PMSE- $\Delta 180$ overexpression compared with control (Fig. 6G, top right), confirmed by the cumulative distribution of the percentage of cells exhibiting varying numbers of neurites (Fig. 6G, bottom). Importantly, the knockdown of GM130 in adult-born DGCs resulted in Golgi dispersion (Fig. $6 H, I$ ) and severe defects in dendrite establishment (Fig. $6 J, K$ ), as with STK25 (Figs. 2, 3) or STRAD knockdown (Fig. 4).

Together, these findings showed that, upon association with the STRAD protein complex, STK25 stabilized a Golgi signaling complex via GM130 recruitment, necessary for both Golgi localization and newborn DGC initial dendrite establishment. We further showed that, in adult-born DGCs expressing the mutated form of STRAD found in patients afflicted by the neurodevelopmental disorder PMSE, this Golgi signaling complex might fail to form, resulting in Golgi dispersion and severe defects in early DGC dendrite establishment.

\section{Discussion}

Our findings showed that asymmetric Golgi positioning regulates the formation of dendrite morphology of the adult-born DGC. Golgi apparatus somatic repositioning, first to the base of a primary neurite pointing to the molecular layer and subsequently to the apical dendrite, might underlie establishment of the dendrite morphology of adult-born DGCs. Mechanistically, STK25- and STRAD-mediated interactions, which stabilized a Golgi signaling complex via GM130 association, were necessary for asymmetric Golgi positioning. Perturbation of this complex caused both Golgi dispersion and severe defects in dendrite establishment of adult-born DGCs.

\section{Single primary dendrite establishment is preceded by extensive neurite remodeling and Golgi repositioning}

A significant fraction of newborn neurons in the subgranular zone of the dentate gyrus in the adult hippocampus integrate into the existing circuit (Altman and Das, 1965; Eriksson et al., 1998; Zhao et al., 2008). Unlike most principal glutamatergic neurons, the mature adult-born DGC exhibits a single dendrite with complex morphology and is devoid of basal dendrites. We showed that establishment of this dendrite morphology was preceded by extensive remodeling of dendrite-like neurites in the neurogenic zone during the first 2 weeks of adult-born DGC integration, as supported by previous studies (Espósito et al., 2005; Zhao et al., 2006).

We determined the extent and timing of early dendritic morphological changes and identified molecular mechanisms that underlie dendrite establishment during adult-born DGC integration. Between 5 and $10 \mathrm{~d}$ of integration, these neurons formed multiple neurites oriented within the GCL or hilus. A small number of mature neurons retain these neurites, either oriented to the hilus (hilar basal dendrites) or the molecular layer (ML) (recurrent basal dendrites) (Ribak et al., 2004; Shapiro et al., 2005), but in most cells, these extraneous neurites are eliminated, resulting in single dendrite establishment. Although the functional importance of neurite remodeling remains unknown, extraneous neurite elimination and single dendrite establishment might be crucial for newborn DGC integration. Thus, Golgi positioning mediated by the protein complex assembled via STK 25 might be crucial for early DGC integration.

We showed that dendrite-like neurite elimination and primary dendrite establishment are associated with dynamic Golgi repositioning to the base of a primary neurite pointing to the ML, once formed, and subsequently to the primary dendrite, while being excluded from other parts of the soma. Golgi repositioning was completed by $10 \mathrm{dpi}$, preceding establishment of the mature dendrite morphology. Thus, Golgi localization to the primary neurite and its exclusion from all other neurites might drive their elimination, leading to single dendrite establishment. In support, genetic manipulations resulting in defective Golgi positioning and its somatic dispersion also resulted in the abnormal persistence of extraneous neurites which demonstrated aberrant Golgi positioning at their base. Golgi dispersion likely did not affect Golgi secretory function, suggesting that Golgi-mediated trafficking might be necessary for extraneous neurites maintenance.

Our findings showed a strong correlation between asymmetric Golgi positioning, neurite elimination, and single dendrite establishment in the GCL. A causal relationship between Golgi localization and dendrite patterning is supported by studies in CA1 pyramidal neurons, where Golgi localization specifies apical versus basal dendrite identities (Horton et al., 2005; Matsuki et al., 2010). In these neurons, the somatic Golgi-apparatus oriented to and post-Golgi secretory trafficking polarized toward the nascent apical dendrite, and the Golgi was required for its growth, maturation, and maintenance (Horton et al., 2005). In pyramidal neurons, upon Golgi localization to the apical dendrite, the Golgi is dispensable for further growth and maturation of basal dendrites, which in these neurons persist throughout life. Conversely, we showed that, during early adult-born DGC integration (5-10 d), the Golgi was required for dendrite-like neurite maintenance. Thus, asymmetric Golgi localization might be selectively required for apical dendrite establishment in principal glutamatergic neurons in general, and as shown here, for single dendrite establishment in newborn DGCs. Moreover, the Golgi complex might be differentially required for basal dendrite maintenance in these neurons.

Selective Golgi complex localization to specific dendrites differs from the distribution of other membrane-bound organelles, including endoplasmic reticulum, endosomes, and mitochondria (Horton et al., 2005), which are found in all dendrites. In adult-born DGCs, the Golgi might regulate extraneous neurite maintenance by directed supply of membrane components (Ye et al., 2007; Zhou et al., 2014) or by serving as acentrosomal microtubule nucleation sites (Ori-McKenney et al., 2012). 


\section{STK25-STRAD-GM130 association mediates Golgi positioning and dendrite establishment}

The Golgi matrix proteins can serve as scaffolds for localization of signaling determinants on Golgi membranes (Barr and Short, 2003; Short and Barr, 2003) and enable asymmetric Golgi positioning to mediate diverse cellular functions, development, migration, or polarization (Kupfer et al., 1982; Kupfer and Dennert, 1984; Bivona et al., 2003; Ríos et al., 2004). The Golgi matrix protein GM130, a crucial regulator of Golgi secretory trafficking (Short and Barr, 2003), acts as such scaffold for asymmetric Golgi positioning (Barr and Short, 2003; Preisinger et al., 2004; Matsuki et al., 2010; Zhou et al., 2014). Our examinations revealed robust expression of the polarity genes STK25 and STRAD, and of GM130, in DCX-positive adult-born DGCs. The kinase STK25 is a crucial regulator of embryonic neuronal polarization upstream of GM130, in a manner that depended on Golgi localization (Matsuki et al., 2010). Furthermore, STK25 acts downstream of the kinase LKB1 and its cofactor STRAD, key regulators of neuronal polarization (Barnes et al., 2007; Shelly et al., 2007; Matsuki et al., 2010).

Structural studies showed cooperative interactions among the STRAD/MO25/LKB1 complex members (Zeqiraj et al., 2009); association of LKB1 with STRAD and MO25 resulted in their mutual stabilization and activation of LKB1 kinase activity, necessary for neuronal polarization (Boudeau et al., 2004; Shelly et al., 2007). We showed that, through association with GM130 and the STRAD/LKB1/MO25 complex, STK25 might form and stabilize a Golgi signaling complex, necessary for Golgi positioning. STK25 showed predominant localization to the base of the primary dendrite, and colocalized with STRAD and GRASP65, indicating association with the Golgi. Furthermore, knockdown of either STK25, STRAD, or GM130 in newborn DGCs caused Golgi dispersion. Last, knockdown of STK25, STRAD, or GM130 resulted in lack of extraneous neurite elimination associated with abnormal Golgi neuritic localization and defects in single dendrite establishment. Although the effects of STK25-STRAD-GM130 manipulations might not be exclusive to the observed effects on the fidelity of the Golgi complex, our findings underscore the role of STK25 as a crucial regulator of dendrite establishment during early newborn DGC integration and show that these effects might be mediated through Golgi positioning. Interestingly, STK25STRAD were expressed in most granule neurons in the GCL of 4- to 6-week-old mouse hippocampus, suggesting that their expression is not restricted to the DCX-positive stage. Whether the STK25 complex is required for later dendritic integration of adult-born DGCs, and whether these effects are mediated via the Golgi complex, remain for future investigation.

\section{STRAD truncation underlying neurodevelopmental disorder PMSE disrupts Golgi positioning and dendrite establishment} The physiological significance for the elaboration of dendrite-like neurites in the immature DGC remains unknown. However, as aberrant hilar or recurrent basal dendrite formation could be involved in the pathogenesis of epilepsy in the dentate gyrus (Spigelman et al., 1998; Ribak et al., 2000; Ribak and Dashtipour, 2002; Dashtipour et al., 2003; Shapiro et al., 2005; Shapiro and Ribak, 2006; Jessberger et al., 2007; Walter et al., 2007; Thind et al., 2008; Scharfman and McCloskey, 2009; Cho et al., 2015), dendrite-like neurite elimination during DGC integration, and the underlying Golgi-associated mechanisms reported here might be crucial for hippocampal circuit formation and function. Interestingly, application of kainic acid (Kaneko et al., 2016), used to induce epilepsy, or culturing embryonic neurons under hyper- excitable conditions (Thayer et al., 2013), caused Golgi fragmentation, suggesting a direct link between the fidelity of Golgi organization and the pathogenesis of epilepsy.

A homozygous mutation in the STRAD gene generating a 180 amino acid C-terminal truncation of the STRAD protein (Puffenberger et al., 2007) underlies the neurodevelopmental disorder PMSE, characterized by cognitive and motor disability and intractable epilepsy. Interestingly, downregulation of STRAD in the embryonic cortex resulted in abnormal cortical development (Orlova et al., 2010; Parker et al., 2013), mediated by aberrant enhanced activation of mammalian target-of-rapamycin complex-1 signaling, regulator of the attenuated temporal maturation and integration of adult-born DGCs (Duan et al., 2007; Kim et al., 2009; Kim et al., 2012; Zhou et al., 2013). We showed that STRADPMSE- $\Delta 180$ interferes with STK25-STRAD-GM130 complex activity in a dominant-negative manner. We found that STRADPMSE- $\Delta 180$ might disable endogenous STK25 from associating with GM130 and STRAD/MO25/LKB1 complex, resulting in GM130-STK25-STRAD Golgi complex disassembly and Golgi dispersion. Consequently, expression of STRAD-PMSE- $\Delta 180$ in newborn DGCs caused severe defects in extraneous neurite elimination and single dendrite establishment, like that with STK25 or STRAD knockdown.

Our findings showed that asymmetric Golgi repositioning mediated via the STK25-STRAD-GM130 complex underlies dendrite establishment of adult-born DGCs during their initial integration. We further showed that STRAD-STK25-GM130 complex disassembly and subsequent Golgi mispositioning might be an important causative factor in PMSE disorder. Aberrant extraneous neurite elimination during early DGC integration and the underlying mechanisms reported here might be crucial for hippocampal circuit formation and function. The Golgi apparatus and associated molecular determinants might therefore be targets for the prevention of abnormal brain development and circuit function, particularly the epilepsy pathology.

\section{References}

Ackermann U, Geering K (1990) Mutual dependence of Na, K-ATPase alpha- and beta-subunits for correct posttranslational processing and intracellular transport. FEBS Lett 269:105-108. CrossRef Medline

Altman J, Das GD (1965) Autoradiographic and histological evidence of postnatal hippocampal neurogenesis in rats. J Comp Neurol 124:319335. CrossRef Medline

Barnes AP, Lilley BN, Pan YA, Plummer LJ, Powell AW, Raines AN, Sanes JR, Polleux F (2007) LKB1 and SAD kinases define a pathway required for the polarization of cortical neurons. Cell 129:549-563. CrossRef Medline

Barr FA, Short B (2003) Golgins in the structure and dynamics of the Golgi apparatus. Curr Opin Cell Biol 15:405-413. CrossRef Medline

Bivona TG, Pérez De Castro I, Ahearn IM, Grana TM, Chiu VK, Lockyer PJ, Cullen PJ, Pellicer A, Cox AD, Philips MR (2003) Phospholipase Cgamma activates Ras on the Golgi apparatus by means of RasGRP1. Nature 424:694698. CrossRef Medline

Boudeau J, Scott JW, Resta N, Deak M, Kieloch A, Komander D, Hardie DG, Prescott AR, van Aalten DM, Alessi DR (2004) Analysis of the LKB1STRAD-MO25 complex. J Cell Sci 117:6365-6375. CrossRef Medline

Cho KO, Lybrand ZR, Ito N, Brulet R, Tafacory F, Zhang L, Good L, Ure K, Kernie SG, Birnbaum SG, Scharfman HE, Eisch AJ, Hsieh J (2015) Aberrant hippocampal neurogenesis contributes to epilepsy and associated cognitive decline. Nat Commun 6:6606. CrossRef Medline

Dashtipour K, Wong AM, Obenaus A, Spigelman I, Ribak CE (2003) Temporal profile of hilar basal dendrite formation on dentate granule cells after status epilepticus. Epilepsy Res 54:141-151. CrossRef Medline

de Anda FC, Pollarolo G, Da Silva JS, Camoletto PG, Feiguin F, Dotti CG (2005) Centrosome localization determines neuronal polarity. Nature 436:704-708. CrossRef Medline

Duan X, Chang JH, Ge S, Faulkner RL, Kim JY, Kitabatake Y, Liu XB, Yang CH, Jordan JD, Ma DK, Liu CY, Ganesan S, Cheng HJ, Ming GL, Lu B, 
Song H (2007) Disrupted-In-Schizophrenia 1 regulates integration of newly generated neurons in the adult brain. Cell 130:1146-1158. CrossRef Medline

Dudek FE, Sutula TP (2007) Epileptogenesis in the dentate gyrus: a critical perspective. In: Progress in brain research (Helen ES, ed), pp 755-773. Amsterdam, the Netherlands: Elsevier.

Eriksson PS, Perfilieva E, Björk-Eriksson T, Alborn AM, Nordborg C, Peterson DA, Gage FH (1998) Neurogenesis in the adult human hippocampus. Nat Med 4:1313-1317. CrossRef Medline

Espósito MS, Piatti VC, Laplagne DA, Morgenstern NA, Ferrari CC, Pitossi FJ, Schinder AF (2005) Neuronal differentiation in the adult hippocampus recapitulates embryonic development. J Neurosci 25:10074-10086. CrossRef Medline

Gao Y, Wang F, Eisinger BE, Kelnhofer LE, Jobe EM, Zhao X (2017) Integrative single-cell transcriptomics reveals molecular networks defining neuronal maturation during postnatal neurogenesis. Cereb Cortex 27: 2064-2077. CrossRef Medline

Ge S, Goh EL, Sailor KA, Kitabatake Y, Ming GL, Song H (2006) GABA regulates synaptic integration of newly generated neurons in the adult brain. Nature 439:589-593. CrossRef Medline

Ge S, Pradhan DA, Ming GL, Song H (2007) GABA sets the tempo for activitydependent adult neurogenesis. Trends Neurosci 30:1-8. CrossRef Medline

Gu Y, Janoschka S, Ge S (2011) Studying the integration of adult-born neurons. J Vis Exp 2011:2548. CrossRef Medline

Horton AC, Rácz B, Monson EE, Lin AL, Weinberg RJ, Ehlers MD (2005) Polarized secretory trafficking directs cargo for asymmetric dendrite growth and morphogenesis. Neuron 48:757-771. CrossRef Medline

Huang W, She L, Chang XY, Yang RR, Wang L, Ji HB, Jiao JW, Poo MM (2014) Protein kinase LKB1 regulates polarized dendrite formation of adult hippocampal newborn neurons. Proc Natl Acad Sci U S A 111:469_ 474. CrossRef Medline

Jareb M, Banker G (1997) Inhibition of axonal growth by brefeldin A in hippocampal neurons in culture. J Neurosci 17:8955-8963. Medline

Jaunin P, Horisberger JD, Richter K, Good PJ, Rossier BC, Geering K (1992) Processing, intracellular transport, and functional expression of endogenous and exogenous alpha-beta $3 \mathrm{Na}, \mathrm{K}$-ATPase complexes in Xenopus oocytes. J Biol Chem 267:577-585. Medline

Jessberger S, Zhao C, Toni N, Clemenson GD Jr, Li Y, Gage FH (2007) Seizure-associated, aberrant neurogenesis in adult rats characterized with retrovirus-mediated cell labeling. J Neurosci 27:9400-9407. CrossRef Medline

Kaneko Y, Sullivan R, Dailey T, Vale FL, Tajiri N, Borlongan CV (2016) Kainic acid-induced Golgi complex fragmentation/dispersal shifts the proteolysis of reelin in primary rat neuronal cells: an in vitro model of early stage epilepsy. Mol Neurobiol 53:1874-1883. CrossRef Medline

Kim JY, Duan X, Liu CY, Jang MH, Guo JU, Pow-anpongkul N, Kang E, Song H, Ming GL (2009) DISC1 regulates new neuron development in the adult brain via modulation of AKT-mTOR signaling through KIAA1212. Neuron 63:761-773. CrossRef Medline

Kim JY, Liu CY, Zhang F, Duan X, Wen Z, Song J, Feighery E, Lu B, Rujescu D, St Clair D, Christian K, Callicott JH, Weinberger DR, Song H, Ming GL (2012) Interplay between DISC1 and GABA signaling regulates neurogenesis in mice and risk for schizophrenia. Cell 148:1051-1064. CrossRef Medline

Kumamoto N, Gu Y, Wang J, Janoschka S, Takemaru K, Levine J, Ge S (2012) A role for primary cilia in glutamatergic synaptic integration of adultborn neurons. Nat Neurosci 15 399- 405:S391. CrossRef Medline

Kupfer A, Dennert G (1984) Reorientation of the microtubule-organizing center and the Golgi apparatus in cloned cytotoxic lymphocytes triggered by binding to lysable target cells. J Immunol 133:2762-2766. Medline

Kupfer A, Louvard D, Singer SJ (1982) Polarization of the Golgi apparatus and the microtubule-organizing center in cultured fibroblasts at the edge of an experimental wound. Proc Natl Acad Sci U S A 79:2603-2607. CrossRef Medline

Matsuki T, Matthews RT, Cooper JA, van der Brug MP, Cookson MR, Hardy JA, Olson EC, Howell BW (2010) Reelin and Stk25 have opposing roles in neuronal polarization and dendritic Golgi deployment. Cell 143:826836. CrossRef Medline

Milburn CC, Boudeau J, Deak M, Alessi DR, van Aalten DM (2004) Crystal structure of MO25 alpha in complex with the $\mathrm{C}$ terminus of the pseudo kinase STE20-related adaptor. Nat Struct Mol Biol 11:193-200. CrossRef Medline
Nakamura N, Rabouille C, Watson R, Nilsson T, Hui N, Slusarewicz P, Kreis TE, Warren G (1995) Characterization of a cis-Golgi matrix protein, GM130. J Cell Biol 131:1715-1726. CrossRef Medline

Ori-McKenney KM, Jan LY, Jan YN (2012) Golgi outposts shape dendrite morphology by functioning as sites of acentrosomal microtubule nucleation in neurons. Neuron 76:921-930. CrossRef Medline

Orlova KA, Parker WE, Heuer GG, Tsai V, Yoon J, Baybis M, Fenning RS, Strauss K, Crino PB (2010) STRADalpha deficiency results in aberrant mTORC1 signaling during corticogenesis in humans and mice. J Clin Invest 120:1591-1602. CrossRef Medline

Osada S, Izawa M, Saito R, Mizuno K, Suzuki A, Hirai S, Ohno S (1997) YSK1, a novel mammalian protein kinase structurally related to Ste20 and SPS1, but is not involved in the known MAPK pathways. Oncogene 14: 2047-2057. CrossRef Medline

Overstreet Wadiche L, Bromberg DA, Bensen AL, Westbrook GL (2005) GABAergic signaling to newborn neurons in dentate gyrus. J Neurophysiol 94:4528-4532. CrossRef Medline

Parker WE, Orlova KA, Parker WH, Birnbaum JF, Krymskaya VP, Goncharov DA, Baybis M, Helfferich J, Okochi K, Strauss KA, et al. (2013) Rapamycin prevents seizures after depletion of STRADA in a rare neurodevelopmental disorder. Sci Transl Med 5:182ra153. CrossRef Medline

Preisinger C, Short B, De Corte V, Bruyneel E, Haas A, Kopajtich R, Gettemans J, Barr FA (2004) YSK1 is activated by the Golgi matrix protein GM130 and plays a role in cell migration through its substrate 14-3-3zeta. J Cell Biol 164:1009-1020. CrossRef Medline

Puffenberger EG, Strauss KA, Ramsey KE, Craig DW, Stephan DA, Robinson DL, Hendrickson CL, Gottlieb S, Ramsay DA, Siu VM, Heuer GG, Crino PB, Morton DH (2007) Polyhydramnios, megalencephaly and symptomatic epilepsy caused by a homozygous 7-kilobase deletion in LYK5. Brain 130:1929-1941. CrossRef Medline

Puthenveedu MA, Bachert C, Puri S, Lanni F, Linstedt AD (2006) GM130 and GRASP65-dependent lateral cisternal fusion allows uniform Golgienzyme distribution. Nat Cell Biol 8:238-248. CrossRef Medline

Ribak CE, Dashtipour K (2002) Neuroplasticity in the damaged dentate gyrus of the epileptic brain. Prog Brain Res 136:319-328. CrossRef Medline

Ribak CE, Tran PH, Spigelman I, Okazaki MM, Nadler JV (2000) Status epilepticus-induced hilar basal dendrites on rodent granule cells contribute to recurrent excitatory circuitry. J Comp Neurol 428:240-253. CrossRef Medline

Ribak CE, Korn MJ, Shan Z, Obenaus A (2004) Dendritic growth cones and recurrent basal dendrites are typical features of newly generated dentate granule cells in the adult hippocampus. Brain Res 1000:195-199. CrossRef Medline

Ríos RM, Sanchís A, Tassin AM, Fedriani C, Bornens M (2004) GMAP-210 recruits gamma-tubulin complexes to cis-Golgi membranes and is required for Golgi ribbon formation. Cell 118:323-335. CrossRef Medline

Scharfman HE, McCloskey DP (2009) Postnatal neurogenesis as a therapeutic target in temporal lobe epilepsy. Epilepsy Res 85:150-161. CrossRef Medline

Shapiro LA, Ribak CE (2006) Newly born dentate granule neurons after pilocarpine-induced epilepsy have hilar basal dendrites with immature synapses. Epilepsy Res 69:53-66. CrossRef Medline

Shapiro LA, Korn MJ, Ribak CE (2005) Newly generated dentate granule cells from epileptic rats exhibit elongated hilar basal dendrites that align along GFAP-immunolabeled processes. Neuroscience 136:823-831. CrossRef Medline

Shelly M, Cancedda L, Heilshorn S, Sumbre G, Poo MM (2007) LKB1/ STRAD promotes axon initiation during neuronal polarization. Cell 129: 565-577. CrossRef Medline

Short B, Barr FA (2003) Membrane traffic: a glitch in the Golgi matrix. Curr Biol 13:R311-R313. CrossRef Medline

Spigelman I, Yan XX, Obenaus A, Lee EY, Wasterlain CG, Ribak CE (1998) Dentate granule cells form novel basal dendrites in a rat model of temporal lobe epilepsy. Neuroscience 86:109-120. CrossRef Medline

Tanabe K, Kani S, Shimizu T, Bae YK, Abe T, Hibi M (2010) Atypical protein kinase $\mathrm{C}$ regulates primary dendrite specification of cerebellar Purkinje cells by localizing Golgi apparatus. J Neurosci 30:16983-16992. CrossRef Medline

Thayer DA, Jan YN, Jan LY (2013) Increased neuronal activity fragments the Golgi complex. Proc Natl Acad Sci U S A 110:1482-1487. CrossRef Medline

Thind KK, Ribak CE, Buckmaster PS (2008) Synaptic input to dentate gran- 
ule cell basal dendrites in a rat model of temporal lobe epilepsy. J Comp Neurol 509:190-202. CrossRef Medline

Tokhtaeva E, Sachs G, Vagin O (2009) Assembly with the Na, K-ATPase alpha(1) subunit is required for export of beta(1) and beta(2) subunits from the endoplasmic reticulum. Biochemistry 48:11421-11431. CrossRef Medline

Tokhtaeva E, Munson K, Sachs G, Vagin O (2010) N-glycan-dependent quality control of the Na, K-ATPase beta(2) subunit. Biochemistry 49: 3116-3128. CrossRef Medline

Walter C, Murphy BL, Pun RY, Spieles-Engemann AL, Danzer SC (2007) Pilocarpine-induced seizures cause selective time-dependent changes to adult-generated hippocampal dentate granule cells. J Neurosci 27:75417552. CrossRef Medline

Yadav S, Puri S, Linstedt AD (2009) A primary role for Golgi positioning in directed secretion, cell polarity, and wound healing. Mol Biol Cell 20: 1728-1736. CrossRef Medline

Yadav S, Linstedt AD (2011) Golgi positioning. Cold Spring Harb Perspect Biol 3:a005322. CrossRef Medline

Ye B, Zhang Y, Song W, Younger SH, Jan LY, Jan YN (2007) Growing den- drites and axons differ in their reliance on the secretory pathway. Cell 130:717-729. CrossRef Medline

Zeqiraj E, Filippi BM, Goldie S, Navratilova I, Boudeau J, Deak M, Alessi DR, van Aalten DM (2009) ATP and MO25alpha regulate the conformational state of the STRADalpha pseudokinase and activation of the LKB1 tumour suppressor. PLoS Biol 7:e1000126. CrossRef Medline

Zhao C, Teng EM, Summers RG Jr, Ming GL, Gage FH (2006) Distinct morphological stages of dentate granule neuron maturation in the adult mouse hippocampus. J Neurosci 26:3-11. CrossRef Medline

Zhao C, Deng W, Gage FH (2008) Mechanisms and functional implications of adult neurogenesis. Cell 132:645-660. CrossRef Medline

Zhou M, Li W, Huang S, Song J, Kim JY, Tian X, Kang E, Sano Y, Liu C, Balaji J, Wu S, Zhou Y, Zhou Y, Parivash SN, Ehninger D, He L, Song H, Ming GL, Silva AJ (2013) mTOR inhibition ameliorates cognitive and affective deficits caused by Discl knockdown in adult-born dentate granule neurons. Neuron 77:647-654. CrossRef Medline

Zhou W, Chang J, Wang X, Savelieff MG, Zhao Y, Ke S, Ye B (2014) GM130 is required for compartmental organization of dendritic Golgi outposts. Curr Biol 24:1227-1233. CrossRef Medline 